\title{
1 Exogenous alginate protects Staphylococcus aureus from killing by Pseudomonas aeruginosa
}

3 Courtney E. Price ${ }^{1}$, Dustin G. Brown², Dominique H. Limoli ${ }^{3}$, Vanessa V. Phelan ${ }^{2}$ and George A.

4 O'Toole ${ }^{1, \#}$

$6{ }^{1}$ Department of Microbiology and Immunology, Geisel School of Medicine at Dartmouth

$7 \quad{ }^{2}$ Department of Pharmaceutical Sciences, Skaggs School of Pharmacy and Pharmaceutical

8 Sciences, University of Colorado, Aurora, CO

$9{ }^{3}$ Department of Microbiology and Immunology, University of lowa, lowa City, IA

12 Running Title: S. aureus $-P$. aeruginosa interactions

14 \#Address correspondence to George A. O’Toole, georgeo@dartmouth.edu

15 Department of Microbiology and Immunology, Geisel School of Medicine at Dartmouth

16 Rm 202 Remsen Building, Hanover, NH 03755

17 Tel: (603) 650-1248

18 Fax: (603) 650-1728

20 Key words: alginate, cystic fibrosis, Pseudomonas aeruginosa, Staphylococcus aureus,

21 polymicrobial, mucoid, Nanostring, PQS, HQNO, pyoverdine, pyochelin, siderophores 
22 ABSTRACT. Cystic fibrosis (CF) patients chronically infected with both Pseudomonas aeruginosa

23 and Staphylococcus aureus have worse health outcomes than patients who are mono-infected

24 with either $P$. aeruginosa or S. aureus. We showed previously that mucoid strains of $P$.

25 aeruginosa can co-exist with S. aureus in vitro due to transcriptional downregulation of several

26 toxic exoproducts typically produced by $P$. aeruginosa, including siderophores, rhamnolipids,

27 and HQNO (2-heptyl-4-hydroxyquinoline N-oxide). Here we demonstrate that exogenous

28 alginate protects $S$. aureus from $P$. aeruginosa in both planktonic and biofilm co-culture models

29 under a variety of nutritional conditions. S. aureus protection in the presence of exogenous

30 alginate is due to transcriptional downregulation of $p v d A$, a gene required for the production of

31 the iron scavenging siderophore pyoverdine, as well as down-regulation of the PQS

32 (Pseudomonas quinolone signal; 2-heptyl-3,4-dihydroxyquinoline) quorum sensing system. The

33 impact of exogenous alginate is independent of endogenous alginate production. We further

34 demonstrate that co-culture of mucoid $P$. aeruginosa with non-mucoid $P$. aeruginosa can

35 mitigate the killing of $S$. aureus by the non-mucoid strain of $P$. aeruginosa, indicating that the

36 mechanism we describe here may function in vivo in the context of mixed infections. Finally, we

37 investigated a panel of mucoid clinical isolates that retain the ability kill S. aureus at late time

38 points, and show that each strain has a unique expression profile, indicating that mucoid

39 isolates can overcome the effects of mucoidy in a strain-specific manner.

41 IMPORTANCE. CF patients are chronically infected by polymicrobial communities of

42 microorganisms. The two dominant bacterial pathogens that infect CF patient lungs are $P$.

43 aeruginosa and S. aureus, with $\sim 30 \%$ of patients co-infected by both species. Patients infected 
44 with both $P$. aeruginosa and S. aureus have worse outcomes than mono-infected patients, and

45 both species persist within the same physical space in the lungs of CF patients. A variety of host

46 and environmental factors have been demonstrated to promote $P$. aeruginosa-S. aureus co-

47 existence, despite evidence that $P$. aeruginosa kills S. aureus when these organisms are co-

48 cultured in vitro. Thus, a better understanding of $P$. aeruginosa-S. aureus interactions,

49 particularly mechanisms by which these microorganisms are able to co-exist in proximal

50 physical space, will lead to better informed treatments for chronic polymicrobial infections.

52 INTRODUCTION

53 Cystic fibrosis (CF) is an autosomal recessive disorder with significant morbidity and

54 mortality affecting $>70,000$ people worldwide $(1,2)$. Decreased function of the cystic fibrosis

55 transmembrane regulator (CFTR), an epithelial cell chloride ion transporter, causes increased

56 viscosity of airway surface liquid and decreased mucociliary clearance of microorganisms in the

57 CF airway $(3,4)$. Over time these microorganisms form complex, polymicrobial communities

58 which are highly tolerant to antibiotic treatment; the airway damage due to these chronic

59 infections eventually leads to respiratory failure (5-15). The development of novel therapeutics

60 have markedly improved patient survival $(2,16-19)$, but despite these recent advances, the

61 progression of CF pulmonary disease is one of chronic infection and inflammation punctuated

62 by periods of clinical exacerbation which cause irreversible damage to lung tissue $(2,8,20-22)$.

63 Pseudomonas aeruginosa and Staphylococcus aureus are the two opportunistic

64 pathogens most commonly isolated from CF patients' lungs (2), and co-infection with both

65 microbes is common $(23,24)$. Co-infection with $P$. aeruginosa and S. aureus alters antibiotic 
tolerance (25-28) and enhances virulence (29) in chronic infection. Furthermore, CF patients

67 who are co-infected with both $P$. aeruginosa and S. aureus have poorer clinical outcomes than those who are mono-infected (23).

Studies of $P$. aeruginosa-S. aureus interactions have demonstrated that $P$. aeruginosa

kills S. aureus in vitro, and this interaction is thought to contribute to the dominance of $P$.

71 aeruginosa over $S$. aureus as CF patients age $(30,31)$. Considerable progress has been made in

72 elucidating the mechanism of $S$. aureus killing mediated by $P$. aeruginosa (32-35). $P$. aeruginosa

73 secretes quorum sensing regulated antimicrobial exoproducts during acute infection, including

74 2-heptyl-4-hydroxyquinoline N-oxide (HQNO), siderophores, rhamnolipids, and phenazines.

75 HQNO and the two P. aeruginosa siderophores, pyoverdine and pyochelin, have been shown to

76 drive $S$. aureus towards a fermentative lifestyle (33), while rhamnolipids disrupt cell membrane

77 integrity $(33,36)$. Phenazines inhibit S. aureus metabolism, as well as play a role in iron

78 acquisition and biofilm development (37-39).

Chronic CF infection is marked by the emergence of mucoid $P$. aeruginosa isolates,

80 which overproduce the exopolysaccharide alginate $(40,41)$. P. aeruginosa virulence towards $S$.

81 aureus is decreased due to the overproduction of alginate, which causes transcriptional

82 downregulation of HQNO, siderophores, and rhamnolipids $(32,33,42)$. The mechanism of

83 alginate overproduction is typically due to mutation in the mucA gene (43-45), a negative

84 regulator of $\sigma^{22}(\mathrm{AlgT} / \mathrm{U})(43,44,46)$. De-repression of $\sigma^{22}$ promotes transcription of many

85 genes, but disruption of $\operatorname{algD}$, the first gene in the alginate biosynthetic operon, restores wild-

86 type levels of antimicrobial exoproducts, demonstrating that alginate production is sufficient 
87 for antimicrobial exoproduct downregulation independent of other transcriptional effects of

$88 \quad \operatorname{AlgT/U}(32)$.

In this study we asked how alginate influences the production of anti-Staphylococcal

90 antimicrobials. We show that exogenous alginate is sufficient to protect S. aureus from $P$.

91 aeruginosa in co-culture, likely via the transcriptional downregulation of several genes whose

92 products are required for $P$. aeruginosa to kill S. aureus. We also show that a three-way co-

93 culture between mucoid and non-mucoid $P$. aeruginosa and $S$. aureus results in attenuated

94 killing of S. aureus, raising the possibility that the mechanism we identified may function in vivo

95 in patients with such mixed infections. Furthermore, the analysis of mucoid isolates of $P$.

96 aeruginosa indicates that some isolates are able to kill S. aureus after prolonged co-culture and

97 that the mechanism of killing is due to diverse transcriptional changes, indicating a dynamic and

98 evolving competition between these two microbes.

\section{RESULTS}

101 Exogenous alginate protects $\boldsymbol{S}$. aureus in co-culture with $\boldsymbol{P}$. aeruginosa. To determine whether

102 the presence of exogenous alginate can promote co-existence between $P$. aeruginosa and $S$.

103 aureus, we investigated a co-culture with 1\% seaweed-derived alginate (Fig. 1A-B; Fig. S1).

104 Cultures were plated onto Pseudomonas Isolation Agar (PIA) and Mannitol Salt Agar (MSA) to

105 quantify colony forming units (CFUs) of $P$. aeruginosa and S. aureus, respectively, over a 10

106 hour co-culture. Because there are minor structural differences between seaweed-derived

107 alginate and $P$. aeruginosa-produced alginate (46), with seaweed derived alginate lacking

108 acetylation, we also isolated alginate from the mucoid $P$. aeruginosa PAO1 mucA22 strain (see 
Materials and Methods) and added this P. aeruginosa PAO1-derived alginate to P. aeruginosa-S.

111 alginate protected S. aureus equally well from killing by P. aeruginosa (Fig. 1A; Fig. S1A). 1\%

112 seaweed-derived alginate did not affect $P$. aeruginosa growth, while $P$. aeruginosa-derived

113 alginate reduced the growth of $P$. aeruginosa by a small but statistically significant amount (Fig.

114 1B; Fig. S1B). We performed several additional experiments which supported our conclusion

115 that the addition of exogenous alginate protects $S$. aureus in planktonic coculture with $P$.

116 aeruginosa by a mechanism other than reduced $P$. aeruginosa growth, and expanded our initial

117 results to demonstrate that a range of exogenous alginate $(0.25 \%-2 \%)$ concentrations protect $S$.

118 aureus from $P$. aeruginosa in planktonic co-culture (Fig. S2-3).

Bacterial interactions can change dramatically under different conditions, so we used a

coculture model previously described by our lab to determine whether exogenous alginate also

121 protects $P$. aeruginosa from $S$. aureus in a biofilm-based coculture model $(25,33)$. After an

122 initial 1-hour attachment phase followed by washing to remove planktonic cells, co-cultures of

123 P. aeruginosa and S. aureus were incubated statically for 16 hours, and then CFUs were

124 enumerated separately for the planktonic and biofilm fractions (see Materials and Methods).

125 Biofilm coculture experiments were performed with P. aeruginosa PAO1 (Fig. 1 C-D; Fig. S3 E-F)

126 and $P$. aeruginosa PA14 (Fig. S3, G-J). S. aureus was killed by $P$. aeruginosa PAO1 in this biofilm

127 model and protected from P. aeruginosa PAO1 with 1\% exogenous alginate in both the biofilm

128 (Fig. 1 C) and planktonic (Fig. 1 D) fraction. P. aeruginosa PAO1 growth was not affected by

129 exogenous alginate in either fraction (Fig. S3, E-F). Results were similar for P. aeruginosa PA14,

130 except that exogenous alginate also modestly boosted $P$. aeruginosa PA14 growth (Fig. S3, I-J). 
131 Experiments with similar results were also performed planktonically with the same minimal

132 media used for biofilm co-culture experiments (Fig. S4). Taken together, these data indicate

133 that exogenous alginate can modulate the killing of $S$. aureus by $P$. aeruginosa, and that

134 exogenous alginate alone is sufficient to protect $S$. aureus from $P$. aeruginosa under a variety of

135 growth conditions.

137 Tri-culture of S. aureus with wild-type and mucoid P. aeruginosa delays S. aureus killing.

138 Because exogenous alginate can modify interactions between $P$. aeruginosa and S. aureus, and

139 CF patients who are co-infected with $P$. aeruginosa and S. aureus are also likely infected with

140 mucoid and non-mucoid $P$. aeruginosa isolates, we hypothesized that mucoid $P$. aeruginosa

141 might affect interactions between wild-type $P$. aeruginosa and S. aureus. We therefore

142 designed a tri-culture experiment where S. aureus was either monocultured, co-cultured with

143 nonmucoid P. aeruginosa PAO1 or mucoid P. aeruginosa PAO1 mucA22, or cultured with both

144 nonmucoid and mucoid P. aeruginosa (Fig. 2; Fig. S5). Each strain of P. aeruginosa was

145 inoculated such that experiments with both mucoid and nonmucoid $P$. aeruginosa strains

146 contained $2 \mathrm{X}$ the initial concentration of $P$. aeruginosa, to ensure that the total concentration

147 of wild-type $P$. aeruginosa remained consistent across all conditions. S. aureus survival

148 increased significantly in both the biofilm and planktonic fractions after 12 hours (Fig. 2A-B) but

149 not after 16 hours (Fig. 2 C-D) of tri-culture with both mucoid and non-mucoid $P$. aeruginosa.

150 These results indicate that mucoid strains can impact interactions between nonmucoid $P$.

151 aeruginosa and S. aureus by delaying S. aureus killing. 
153 Alginate synthesis is not required for protection by exogenous alginate. We hypothesized

154 that exogenous alginate might protect $S$. aureus by stimulating $P$. aeruginosa to produce

155 alginate and therefore lower virulence towards S. aureus via known changes that accompany

156 mucoidy (32). We used a $P$. aeruginosa strain that cannot synthesize alginate, $P$. aeruginosa

157 PAO1 algD::FRT, to test whether the ability to self-produce alginate is required for $S$. aureus

158 protection by exogenous alginate. Using the biofilm co-culture model, we measured a

159 significant increase in S. aureus survival in the presence of $P$. aeruginosa PAO1 algD::FRT with

160 exogenous alginate in both the biofilm and planktonic fractions (Fig. 3 A-B). Growth of $P$.

161 aeruginosa PAO1 and P. aeruginosa PAO1 algD::FRT was non-significantly and significantly

162 increased, respectively, by exogenous alginate in the biofilm fraction only (Fig. 3 C-D).

163 Therefore, the ability to self-produce alginate is not required to reduce virulence towards $S$.

164 aureus in the presence of exogenous alginate.

165

166 Exogenous alginate transcriptionally decreases siderophore production by $\boldsymbol{P}$. aeruginosa. S.

167 aureus is protected from mucoid $P$. aeruginosa due to the transcriptional downregulation of

168 genes in the mucoid strain of $P$. aeruginosa required to produce the iron chelator pyoverdine,

169 rhamnolipid surfactant and the redox active molecule HQNO (32). Thus, we investigated

170 whether exogenous alginate might function via a similar mechanism. Pyoverdine production

171 was quantified by measuring relative fluorescence units (RFUs) with excitation at 400 and

172 absorbance at 460 of supernatant from $P$. aeruginosa grown with $+/-1 \%$ exogenous alginate. $P$.

173 aeruginosa PAO1 mucA22 and P. aeruginosa PAO1 $\triangle p v d A$ were included as controls.

174 Pyoverdine decreased significantly when P. aeruginosa PAO1 was cultured with exogenous 
175 alginate (Fig. 4A). Interestingly, while mucoid P. aeruginosa PAO1 mucA22 produced

176 significantly less pyoverdine than wild-type $P$. aeruginosa PAO1, addition of exogenous alginate

177 further decreased pyoverdine production by the mucoid strain.

We performed a quenching experiment to test whether exogenous alginate can absorb

179 pyoverdine from the media or mask its signal in our assay. P. aeruginosa PAO1 supernatants

180 were diluted $1 / 2 \mathrm{X}$ with DI water or $2 \%$ exogenous alginate and pyoverdine was quantified after

181 an additional 5 minute and 24 hour incubation at $37^{\circ} \mathrm{C}$. There was a small but significant and

182 repeatable decrease in total pyoverdine measured when supernatants were diluted in alginate

183 versus water (Fig. 4B), indicating that alginate may absorb a small portion of the pyoverdine

184 present or partially mask its signal in our assay.

To test whether pyoverdine production is reduced at the transcriptional level by

exogenous alginate, we cultured $P$. aeruginosa PAO1 in the presence and absence of exogenous

187 alginate and measured transcription of $p v d A$, a gene essential for pyoverdine production, by

188 qRT-PCR. The mucoid strain $P$. aeruginosa PAO1 mucA22 was used as a control, as this strain

189 transcriptionally downregulates $p v d A$ (32). The expression of $p v d A$ was significantly reduced

190 when P. aeruginosa PAO1 was grown with 1\% exogenous alginate (Fig. 4C). Consistent with

191 previous data (32), pvdA expression was also decreased in the mucoid strain carrying the

192 mucA22 mutation. When pyoverdine production is low, $P$. aeruginosa will typically increase the

193 production of pyochelin, the other major $P$. aeruginosa siderophore, so we used liquid

194 chromatography tandem mass spectrometry (LC-MS/MS) to quantify pyochelin. P. aeruginosa

195 PAO1 also reduced pyochelin production upon exposure to exogenous alginate (Fig. 4D). Taken

196 together, these results indicate that exogenous alginate reduces the production of both $P$. 
aeruginosa siderophores pyoverdine and pyochelin, likely via transcriptional down regulation, and may also at least partially sequester siderophores that are produced.

\section{Exogenous alginate post-transcriptionally decreases rhamnolipid production by $P$.}

aeruginosa. Rhamnolipids are surfactants produced by $P$. aeruginosa that intercalate into the

cytoplasmic membrane of $S$. aureus (47). Surfactants disrupt the surface tension of liquids, a rhamnolipid-deficient strain $P$. aeruginosa PAO1 rhlA::Gm was not affected by the presence of exogenous alginate (Fig. 5B).

Because the drop collapse assay is an estimate of total rhamnolipids, we also used LCrhamnolipid quantity significantly decreased by $50 \%$ with the addition of exogenous alginate

211 when measured by this more sensitive method (Fig. 5C). The fold-change decrease was similar

212 for both mono- and di- rhamnolipids (Fig. 5D). To determine whether the reduced rhamnolipid

213 production was due to transcriptional regulation, we measured the relative expression of $r h / A$

214 for $P$. aeruginosa PAO1 +/- 1\% alginate, and $P$. aeruginosa PAO1 mucA22. Transcription of $r$ IA

215 was not significantly different between P. aeruginosa PAO1 +/- 1\% exogenous alginate (Fig. 5E).

217 Exogenous alginate downregulates PQS quorum sensing. The PQS (Pseudomonas quinolone

218 signal; 2-heptyl-3,4-dihydroxyquinoline) quorum sensing system, which impacts a range of $P$. 
aeruginosa virulence factors (Fig. 6A), is known to be down-regulated by mucoid $P$. aeruginosa strains $(32,48)$. We therefore measured the impact of exogenous alginate on several products

221 regulated by the PQS quorum sensing system, with $P$. aeruginosa mucA22 as a control.

222 Interestingly, both self-produced and exogenous alginate decrease production of several key

223 PQS-regulated products, including HQNO, HHQ (4-hydroxy-2-heptylquinoline), and PQS (Fig. 6B-

224 D) as well as downstream factors such as phenazines PCA (phenazine-1-carboxylic acid) and

225 pyocyanin (Fig. 6E-F).

226

227 Expression profiles of $\boldsymbol{P}$. aeruginosa in the presence of exogenous alginate. To determine how

228 exogenous alginate impacts $P$. aeruginosa gene expression more generally, we used the

229 Nanostring PAV2 codeset (49) to compare $P$. aeruginosa gene expression in the presence and

230 absence of exogenous alginate and S. aureus. Nansotring is a digital multiplexed technology for

231 direct quantification of RNA transcripts. The PAV2 codeset used in this study contains probes

232 for 75 transcripts associated with $P$. aeruginosa genes known or suspected to be expressed in

233 the CF airway (49). P. aeruginosa PAO1 and P. aeruginosa PA14 were cultured to mid-log phase

234 and then sub-cultured into fresh media +/- 1\% alginate. Samples were collected after an

235 additional 45 minutes of growth and expression was analyzed by Nanostring according to the

236 manufacturer's protocols.

237 Ten genes were significantly differentially regulated by $P$. aeruginosa PAO1 in the

238 presence of exogenous alginate (Fig. 7A). We previously used the same Nanostring codeset to

239 compare mucoid and nonmucoid $P$. aeruginosa PAO1 gene expression, and measured a

240 significant downregulation of $p v d A, \operatorname{rhl} A, p c h C$, flic type $B$, and nor $C$, as well as a significant 
241 upregulation of $a l g l$ and $a l g D$. Of these genes, only $p c h C$ is significantly differentially regulated

242 in the same direction across both studies, indicating that transcriptional changes due to

243 exogenous alginate are not broadly similar to the effects of self-produced alginate by $P$.

244 aeruginosa PAO1. We observed an overlap in significant transcriptional changes in response to

245 exogenous alginate between $P$. aeruginosa PA14 and P. aeruginosa PAO1 for five genes - cyoA,

246 narG, norC, pchC, and pscC (Fig. 7B). P. aeruginosa PA14 also had a large number of genes with

247 a small but significant transcriptional upregulation in the presence of exogenous alginate

248 (Supplemental Tables 1-2).

249

To understand how longer exposure to exogenous alginate and the presence of S. aureus

250 effect $P$. aeruginosa transcription, we cultured $P$. aeruginosa PA14 for 8 hours $+/-0.25 \%$

251 alginate and +/- S. aureus. Nanostring counts were normalized and analyzed as previously

252 described (Supplemental Tables 3-4). Only three genes were significantly differentially

253 expressed by $P$. aeruginosa PA14 monocultured in the presence of alginate relative to $P$.

254 aeruginosa PA14 monocultured without alginate (nirS, norC, and popB; Fig. 7C). When $P$.

255 aeruginosa PA14 was co-cultured with S. aureus the same three genes had altered expression,

256 as well as ercS, exoU, fliC type B, and lasB (Fig. 7D). Notably, fliC type $B$ is also downregulated by

257 mucoid $P$. aeruginosa relative to wild-type $P$. aeruginosa (32). The data was further analyzed by

258 clustering and principal component analysis, with results supporting that S. aureus has a larger

259 effect on overall gene expression than exogenous alginate (Fig. S6).

$260 \quad$ We hypothesized that some of the genes transcriptionally downregulated by $P$.

261 aeruginosa in the presence of exogenous alginate might contribute to $S$. aureus protection in

262 coculture. We focused on downregulated genes because the Nanostring codeset genes are 
263 related to virulence and pathogenesis, making it unlikely that upregulation of any of these

264 genes would positively impact S. aureus survival. We utilized a P. aeruginosa PA14

265 nonredundant transposon mutant library (50) to test whether any of these factors had a direct

266 impact on S. aureus survival. We performed a biofilm coculture experiment for $P$. aeruginosa

267 PA14 mutants of each gene identified as significantly down regulated in any Nanostring

268 experiment and found that disruption of exoT, flic, or pqsE significantly enhanced S. aureus

269 survival in the biofilm fraction in the presence of $P$. aeruginosa PA14 (Fig. 7E). The fliC and pqsE

270 mutants also enhanced S. aureus survival in the planktonic fraction (Fig. 7F). Only fliC altered $P$.

271 aeruginosa PA14 growth, which was slightly but significantly increased in the biofilm fraction

272 (Fig. S7 A-B).

Because flic mutants do not produce flagella, we suspected functional flagella might be

274 required for S. aureus killing. We therefore tested flgK and motABCD mutants in the biofilm co-

275 culture model and saw that these mutations also prevented S. aureus killing by P. aeruginosa

276 PA14 (Fig. 7 G-H) without disrupting P. aeruginosa PA14 growth (Fig. S7 C-D). Because flgK

277 mutants are missing the flagellar cap and mot $A B C D$ mutants produce a nonfunctional flagellum,

278 these findings indicate that flagellar function is required for full virulence of $P$. aeruginosa

279 towards S. aureus, and furthermore, suggest that both self-produced and exogenous alginate

280 reduce $P$. aeruginosa-mediated virulence towards S. aureus, in part, by downregulating flagellar

281 genes. 
aureus when cocultured in vitro. Interestingly, other previous work published by our lab

286 indicated that at least one mucoid clinical isolate, P. aeruginosa FRD1, can kill S. aureus in co-

287 culture (33). To determine whether other mucoid clinical isolates could kill S. aureus during in

288 vitro co-culture, in part to understand the basis by which these "mucoid killers" could function,

289 we selected a panel of mucoid clinical isolates identified in previous studies (32) to co-culture

290 with S. aureus.

We co-cultured $P$. aeruginosa clinical isolates with $S$. aureus JE2 and enumerated the

292 viability of S. aureus out to 24 hours of co-culture due to the slow growth of some of the clinical

293 isolates relative to laboratory strains. The ability of mucoid clinical isolates to kill S. aureus in

294 coculture varies by strain (Fig. 8A), with some strains such as FRD1 killing robustly, while others

295 such as CFBRPA33 showing no killing of S. aureus. Mucoidy does still provide some protection

296 from S. aureus even at late time points, as non-mucoid revertants for FRD1 and Cl2224 killed S.

297 aureus more efficiently than the mucoid strains at 16 and 24 hours. We quantified reversion

298 rates for $P$. aeruginosa FRD1 and $P$. aeruginosa CFBRPA32 to determine whether killing is due

299 to high rates of reversion to non-mucoid phenotypes, and observed reversion rates $<0.1 \%$ after

30024 hours of culture (Supplemental Table 5).

$301 \quad$ We used the Nanostring codeset described in the previous section to generate

302 expression data for P. aeruginosa PAO1, CFBRPA32 (a "mucoid killer"), and the mucoid

303 laboratory strain P. aeruginosa PAO1 mucA22 after 24 hours of growth. Nanostring counts were

304 normalized as described previously and then analyzed by two-way ANOVA followed by Tukey's

305 post-test between all three strains (Supplemental Tables 6-7). Of the 75 transcripts measured, 
seventeen genes were significantly differentially regulated between at least one pair of strains

307 (Fig. 8B). profile of CFBRPA32 is more similar to that of the mucoid laboratory strain P. aeruginosa PAO1 mucA22 than nonmucoid $P$. aeruginosa PAO1 (Fig. 8B). However, eight transcripts were

311 significantly differentially regulated between $P$. aeruginosa CFBRPA32 and $P$. aeruginosa PAO1

312 mucA22: pqsE, pqsA, pchC, phzC1/2, fliC type $A$, fliC type $B$, ercS, and pldA (Fig. 8C). Increased

313 expression of $p q s E, p q s A$ and $p c h C$ indicates a potential role for both quorum sensing and iron

314 acquisition in the ability of CFBRPA32 to kill S. aureus. CFBRPA32 pqsE expression is $>50$-fold

315 higher than P. aeruginosa PAO1 mucA22 and >125-fold higher than P. aeruginosa PAO1.

316 Because pqsE is essential for $P$. aeruginosa killing of $S$. aureus, high expression may contribute

317 to the ability of $P$. aeruginosa CFBRPA32 to kill S. aureus at late time points.

318 We chose to perform our Nanostring experiment with CFBRPA32 because there is

319 publicly available Nanostring data (49) for several other mucoid clinical isolates we examined

320 (CFRL8, Cl2224, Cl228, and FRD1). We re-analyzed this data to determine whether we could

321 identify a shared expression pattern between mucoid killers (Fig. S8). However, there was no

322 individual gene other than algD that correlated with S. aureus survival. Taken together, our

323 data indicate that mucoid $P$. aeruginosa strains with the ability to kill S. aureus in co-culture

324 likely do so via strain-specific mechanisms, and furthermore, some of the genes identified in

325 our analysis of "mucoid killers" overlap with functions down-regulated by exogenous alginate. 


\section{Discussion} microorganisms, the host immune system, and nutrient availability all contribute to a complex and dynamic environment $(8,13,31)$. Here we demonstrate that alginate, which is known to

332 impact both host-pathogen and pathogen-pathogen interactions, also indirectly impacts

333 pathogen-pathogen interactions $(51,52)$. Protection of $S$. aureus from $P$. aeruginosa by

334 exogenous alginate is consistent across a variety of nutritional and physiological contexts, given

335 that protection occurs in liquid and biofilm modes of growth, and in both rich and minimal

336 media with two strains of P. aeruginosa (PAO1 and PA14; Fig. 1; Fig. S1-S4). Furthermore,

337 alginate production by a mucoid strain of $P$. aeruginosa is sufficient to delay $S$. aureus killing by

338 a nonmucoid strain of $P$. aeruginosa when all three strains are cultured together (Fig. 2; Fig. S5),

339 demonstrating the potential for mucoid strains to alter interactions between non-mucoid $P$.

340 aeruginosa and S. aureus strains in vivo.

While $P$. aeruginosa robustly kills $S$. aureus in vitro, it has been demonstrated that

342 reduction of any single virulence factor will greatly diminish the ability of $P$. aeruginosa to kill $S$.

343 aureus. We demonstrate that production of $P$. aeruginosa siderophores, pyoverdine and

344 pyochelin, is lowered when $P$. aeruginosa is cultured in exogenous alginate and that this

345 decrease is due at least in part to transcriptional downregulation of pvdA (Fig. 4). Notably, the

346 decrease in siderophore production in the presence of exogenous alginate may not be solely

347 due to transcriptional changes. The incubation of supernatant from wild-type $P$. aeruginosa

348 with alginate reduces total pyoverdine levels, indicating a possible role for sequestration of

349 siderophores by alginate (Fig. 4A-B). Exogenous alginate, like self-produced alginate, causes 
350 transcriptional downregulation of $p v d A$ expression, indicating that self-produced alginate may

351 also lead to decreased expression of this gene via an outside-in signaling mechanism. There is

352 precedence for exogenous exopolysaccharides regulating transcription, as the

353 exopolysaccharide Psl is known to act as an extracellular signal to increase biofilm formation by

354 stimulating c-di-GMP synthesis, although the mechanism is still unknown (53). We also

355 demonstrate a modest decrease in total rhamnolipids across multiple culture methods which is

356 not dependent on transcriptional regulation (Fig. 5). However, it is unclear whether this modest

357 rhamnolipid decrease is enough to affect S. aureus survival. Exogenous alginate also interferes

358 with $P$. aeruginosa quorum sensing, as demonstrated by the decrease in levels of HQNO, AQs,

359 and phenazines (Fig. 6). The combined interference with HQNO, siderophore, and phenazine

360 production all likely contributes to $S$. aureus survival in the presence of exogenous alginate.

361 Finally, we demonstrate that a functional flagellum is required for S. aureus killing. While

362 flagella are considered virulence factors in the host-pathogen context, their role in $P$.

363 aeruginosa-S. aureus interactions has not previously been demonstrated. Furthermore, this

364 demonstrates that exogenous alginate has the capacity, at least in part, to affect $P$. aeruginosa

365 strains in a manner analogous to self-produced alginate, that is by reducing production of anti-

366 Staphylococcal factors. An interesting question that remains is whether the response of $P$.

367 aeruginosa to exogenous alginate is specific; it is possible that the mechanism by which $P$.

368 aeruginosa senses and responds to alginate is due, for example, to increased viscosity.

369 Alternatively, alginate could be enforcing spatial structure or influencing S. aureus gene

370 expression. 
Interestingly, the presence of alginate can be overcome in some mucoid clinical isolates

372 through unique re-wiring of gene expression (Fig. 8), with the apparent increased expression of

373 genes coding for one or more key anti-Staphylococcal factors. Thus, endogenous and

374 exogenous alginate seem to impact an overlapping set of functions that modulate the

375 interaction between $P$. aeruginosa and S. aureus. Our observations are not entirely surprising

376 in the context of the evolving nature of both P. aeruginosa and S. aureus within the CF lung.

377 Finally, our study highlights the importance of the physical environment within the CF lung on

378 the outcomes of polymicrobial interactions, and the need for further studies examining how

379 different specific aspects of the CF lung environment can impact both polymicrobial

380 interactions and overall patient outcomes.

Materials and Methods

383 Detailed protocols and strains used in this study are available in the supplemental text.

384 Media. All experiments were performed in tryptic soy broth (TSB) or MEM. MEM was

385 supplemented with $2 \mathrm{mM} \mathrm{L-glutamine} \mathrm{(L-gln)} \mathrm{+/-} \mathrm{0.4 \%} \mathrm{L-arginine} \mathrm{(L-arg).} \mathrm{For} \mathrm{experiments}$

386 containing alginate, MEM was diluted $1 / 2 \mathrm{X}$ with sterile DI water or alginate. Seaweed alginate

387 was prepared by dissolving alginic acid sodium salt (Sigma) in DI water and autoclaving to

388 sterilize.

389 Cocultures. Overnight cultures were diluted to a final concentration of 0.05 OD. For

390 experiments in MEM, strains were centrifuged and resuspended in MEM L-GIn prior to dilution.

391 Strains in flasks were grown shaking at $225 \mathrm{rpm}$ at $37^{\circ} \mathrm{C}$, and tubes were placed on a culture

392 wheel at $37^{\circ} \mathrm{C} .100 \mathrm{uL}$ total volume was inoculated in a plastic 96 -well plate for biofilm cultures. 
393 At indicated time points, culture samples were serially diluted in PBS, and plated onto

394 Pseudomonas Isolation Again (PIA) or Mannitol Salt Agar (MSA) to quantify viable P. aeruginosa

395 and S. aureus, respectively.

396 Alginate preparation and quantification. P. aeruginosa PAO1 mucA22 was cultured in $25 \mathrm{~mL}$

397 TSB overnight shaking at $225 \mathrm{RPM}$ and $37^{\circ} \mathrm{C}$ and alginate was isolated as previously described

398 (32), with minor modifications detailed in the supplemental methods. The concentration of

399 alginate in solution was determined by the carbazole method described by Knutson and Jeanes

400 (54).

401 Siderophore quantification. Relative fluorescence of supernatants were quantified at 400nm

402 excitation and 460nm emission. Measurements were normalized to CFU/mL.

403 Drop collapse assay. Supernatants were serially diluted $1 / 2 \mathrm{X}$ in PBS and were scored as the

404 reciprocal of the highest dilution at which the drop collapsed. Scores were normalized to

$405 \mathrm{CFU} / \mathrm{mL}$.

406 q-RT-PCR. Cell cultures were pelleted (centrifuge $2 \mathrm{~min}, 14,000 \mathrm{rpm}$ at $4^{\circ} \mathrm{C}$ ) and immediately

407 frozen in ethanol cooled with dry ice. RNA was isolated with TRIzol (Zymo Research) as

408 described by the manufacturer. DNA was removed by three sequential treatments with Turbo-

409 DNAse treatment (Invitrogen), cDNA was prepared (RevertAid First Strand cDNA synthesis kit),

410 and qRT-PCR was performed with SsoFast EvaGreen Supermix (BioRad).

411 LC-MS/MS. Detailed descriptions of LC-MS/MS supernatant extraction, analysis, feature finding,

412 and molecular annotation are available in the supplemental text.

413 Nanostring. Total RNA was prepared as described for q-RT-PCR. The PAV2 codeset (49)

414 was incubated with total RNA as described by the manufacturer's protocol. 
416 Acknowledgements. This work was supported by_NIH Grant R37 Al83256-06 to G.A.O and

417 T32AI007363 to C.E.P. Additional support was provided by the CF-Research Development

418 Program (STANTO07R0), DartCF and the BBC (P30-DK117469). The Phelan lab is supported by

419 the ALSAM Foundation (L.S. Skaggs Professorship and Therapeutic Innovation Award) and the

420 NIH (R35 GM128690-01). We thank D.A. Hogan for providing access to clinical isolates, AE

421 Baker for PA14 $\Delta$ fliC, and Tom Hampton for providing advice on expression data analysis.

422 Clinical CF isolates CFBRPA32 and CFBRPA33 were provided by the CF Biospecimen Registry at

423 the Children's Healthcare of Atlanta and Emory University CF Discovery Core. 


\section{Literature Cited.}

429 1. Cutting GR. 2015. Cystic fibrosis genetics: from molecular understanding to clinical

$430 \quad$ application. Nat Rev Genet 16:45-56.

$4312 . \quad$ Cystic Fibrosis Foundation. 2017 Patient registry annual data report.

432 3. Riordan JR, Rommens JM, Kerem B, Alon N, Rozmahel R, Grzelczak Z, Zielenski J, Lok S,

433 Plavsic N, Chou JL. 1989. Identification of the cystic fibrosis gene: cloning and

$434 \quad$ characterization of complementary DNA. Science 245:1066-73.

435 4. Kurbatova P, Bessonov N, Volpert V, Tiddens HAWM, Cornu C, Nony P, Caudri D, CRESim

$436 \quad$ Working Group. 2015. Model of mucociliary clearance in cystic fibrosis lungs. J Theor Biol

$437 \quad 372: 81-88$.

438 5. Rogers GB, Hart CA, Mason JR, Hughes M, Walshaw MJ, Bruce KD. 2003. Bacterial

439 diversity in cases of lung infection in cystic fibrosis patients: 16S ribosomal DNA (rDNA)

$440 \quad$ length heterogeneity PCR and 16S rDNA terminal restriction fragment length

$441 \quad$ polymorphism profiling. J Clin Microbiol 41:3548-58.

442 6. Bittar F, Richet H, Dubus J-C, Reynaud-Gaubert M, Stremler N, Sarles J, Raoult D, Rolain J-

443 M. 2008. Molecular detection of multiple emerging pathogens in sputa from cystic

$444 \quad$ fibrosis patients. PLoS One 3:e2908.

445 7. Sibley CD, Rabin H, Surette MG. 2006. Cystic fibrosis: a polymicrobial infectious disease.

$446 \quad$ Future Microbiol 1:53-61.

447 8. Filkins LM, O’Toole GA. 2015. Cystic fibrosis lung infections: polymicrobial, complex, and $448 \quad$ hard to treat. PLoS Pathog 11:e1005258.

449 9. Zhao J, Schloss PD, Kalikin LM, Carmody LA, Foster BK, Petrosino JF, Cavalcoli JD, 
VanDevanter DR, Murray S, Li JZ, Young VB, LiPuma JJ. 2012. Decade-long bacterial community dynamics in cystic fibrosis airways. Proc Natl Acad Sci 109:5809-5814.

452 10. Moreau-Marquis S, Stanton BA, O’Toole GA. 2008. Pseudomonas aeruginosa biofilm formation in the cystic fibrosis airway. Pulm Pharmacol Ther 21:595-599.

454 11. Peters BM, Jabra-Rizk MA, O’May GA, Costerton JW, Shirtliff ME. 2012. Polymicrobial interactions: impact on pathogenesis and human disease. Clin Microbiol Rev 25:193-213.

456 12. Hogan DA, Willger SD, Dolben EL, Hampton TH, Stanton BA, Morrison HG, Sogin ML, Czum J, Ashare A. 2016. Analysis of lung microbiota in bronchoalveolar lavage, protected brush and sputum samples from subjects with mild-To-moderate cystic fibrosis lung disease. PLoS One 11:e0149998.

13. de Koff EM, Groot KM de W, Bogaert D. 2016. Development of the respiratory tract microbiota in cystic fibrosis. Curr Opin Pulm Med 22:623-628.

14. Leclair LW, Hogan DA. 2010. Mixed bacterial-fungal infections in the CF respiratory tract. Med Mycol 48:S125-S132.

464 15. Lynch S V., Bruce KD. 2013. The cystic fibrosis airway microbiome. Cold Spring Harb Perspect Med 3:a009738-a009738.

466 16. Mogayzel PJ, Naureckas ET, Robinson KA, Mueller G, Hadjiliadis D, Hoag JB, Lubsch L, health. Am J Respir Crit Care Med 187:680-9.

470 17. Wainwright CE, Elborn JS, Ramsey BW. 2015. Lumacaftor-Ivacaftor in patients with cystic 471 fibrosis homozygous for phe508del CFTR. N Engl J Med 373:1783-4. 
472 18. Van Goor F, Hadida S, Grootenhuis PDJ, Burton B, Cao D, Neuberger T, Turnbull A, Singh

477 19. Saiman L, Siegel JD, LiPuma JJ, Brown RF, Bryson EA, Chambers MJ, Downer VS, Fliege J, Society for Healthcare Epidemiology of America. 2014. Infection prevention and control

20. Sanders DB, Bittner RCL, Rosenfeld M, Redding GJ, Goss CH. 2011. Pulmonary with cystic fibrosis. Pediatr Pulmonol 46:393-400.

21. Sanders DB, Bittner RCL, Rosenfeld M, Hoffman LR, Redding GJ, Goss CH. 2010. Failure to J Respir Crit Care Med 182:627-632.

22. O’Sullivan BP, Freedman SD. 2009. Cystic fibrosis. Lancet (London, England) 373:1891-

490 23. Limoli DH, Yang J, Khansaheb MK, Helfman B, Peng L, Stecenko AA, Goldberg JB. 2016. 
494 24. Limoli DH, Hoffman LR. 2019. Help, hinder, hide and harm: what can we learn from the interactions between Pseudomonas aeruginosa and Staphylococcus aureus during respiratory infections? Thorax thoraxjnl-2018-212616.

497

25. Orazi G, O’Toole GA. 2017. Pseudomonas aeruginosa alters Staphylococcus aureus sensitivity to vancomycin in a biofilm model of cystic fibrosis Infection. MBio 8.

26. DeLeon S, Clinton A, Fowler H, Everett J, Horswill AR, Rumbaugh KP. 2014. Synergistic interactions of Pseudomonas aeruginosa and Staphylococcus aureus in an in vitro wound model. Infect Immun 82:4718-28.

27. Beaudoin T, Yau YCW, Stapleton PJ, Gong Y, Wang PW, Guttman DS, Waters V. 2017. tobramycin resistance. NPJ Biofilms Microbiomes 3:25.

28. Radlinski L, Rowe SE, Kartchner LB, Maile R, Cairns BA, Vitko NP, Gode CJ, Lachiewicz AM, Wolfgang MC, Conlon BP. 2017. Pseudomonas aeruginosa exoproducts determine antibiotic efficacy against Staphylococcus aureus. PLOS Biol 15:e2003981.

29. Alves PM, Al-Badi E, Withycombe C, Jones PM, Purdy KJ, Maddocks SE. 2018. Interaction between Staphylococcus aureus and Pseudomonas aeruginosa is beneficial for colonisation and pathogenicity in a mixed biofilm. Pathog Dis 76.

511 30. Pernet E, Guillemot L, Burgel P-R, Martin C, Lambeau G, Sermet-Gaudelus I, Sands D, aeruginosa eradicates Staphylococcus aureus by manipulating the host immunity. Nat Commun 5:5105.

515 31. Nguyen AT, Oglesby-Sherrouse AG. 2016. Interactions between Pseudomonas aeruginosa 
and Staphylococcus aureus during co-cultivations and polymicrobial infections. Appl Microbiol Biotechnol 100:6141-6148.

518 32. Limoli DH, Whitfield GB, Kitao T, Ivey ML, Davis MR, Grahl N, Hogan DA, Rahme LG, Howell PL, O’Toole GA, Goldberg JB. 2017. Pseudomonas aeruginosa alginate overproduction promotes coexistence with Staphylococcus aureus in a model of cystic fibrosis respiratory infection. MBio 8.

522 33. Filkins LM, Graber JA, Olson DG, Dolben EL, Lynd LR, Bhuju S, O'Toole GA. 2015. towards fermentative metabolism and reduced viability in a cystic fibrosis model. J Bacteriol 197:2252-64.

34. Rüger M, Ackermann M, Reichl U. 2014. Species-specific viability analysis of Pseudomonas aeruginosa, Burkholderia cepacia and Staphylococcus aureus in mixed culture by flow cytometry. BMC Microbiol 14:56.

35. Nguyen AT, Jones JW, Ruge MA, Kane MA, Oglesby-Sherrouse AG. 2015. Iron depletion enhances production of antimicrobials by Pseudomonas aeruginosa. J Bacteriol

36. Bharali P, Saikia JP, Ray A, Konwar BK. 2013. Rhamnolipid (RL) from Pseudomonas aeruginosa OBP1: a novel chemotaxis and antibacterial agent. Colloids Surf B Biointerfaces 103:502-9.

535 37. Wang Y, Wilks JC, Danhorn T, Ramos I, Croal L, Newman DK. 2011. Phenazine-1carboxylic acid promotes bacterial biofilm development via ferrous iron acquisition. J 
538 38. Hotterbeekx A, Kumar-Singh S, Goossens H, Malhotra-Kumar S. 2017. In vivo and In vitro

interactions between Pseudomonas aeruginosa and Staphylococcus spp. Front Cell Infect

39. Noto MJ, Burns WJ, Beavers WN, Skaar EP. 2017. Mechanisms of pyocyanin toxicity and genetic determinants of resistance in Staphylococcus aureus. J Bacteriol 199.

40. Parad RB. 1996. Heterogeneity of phenotype in two cystic fibrosis patients homozygous for the CFTR exon 11 mutation G551D. J Med Genet 33:711-3.

41. Schurr MJ, Yu H, Martinez-Salazar JM, Boucher JC, Deretic V. 1996. Control of AlgU, a member of the sigma E-like family of stress sigma factors, by the negative regulators MucA and MucB and Pseudomonas aeruginosa conversion to mucoidy in cystic fibrosis. J Bacteriol 178:4997-5004.

42. Baldan R, Cigana C, Testa F, Bianconi I, De Simone M, Pellin D, Di Serio C, Bragonzi A, influences virulence of Staphylococcus aureus in vitro and murine models of co-infection.

553 43. Martin DW, Schurr MJ, Mudd MH, Govan JR, Holloway BW, Deretic V. 1993. Mechanism of conversion to mucoidy in Pseudomonas aeruginosa infecting cystic fibrosis patients.

44. Bragonzi A, Wiehlmann L, Klockgether J, Cramer N, Worlitzsch D, Doring G, Tummler B. patients with cystic fibrosis. Microbiology 152:3261-3269. 
fibrosis: characterization of muc mutations in clinical isolates and analysis of clearance in a mouse model of respiratory infection. Infect Immun 65:3838-46.

46. Ramsey DM, Wozniak DJ. 2005. Understanding the control of Pseudomonas aeruginosa alginate synthesis and the prospects for management of chronic infections in cystic fibrosis. Mol Microbiol 56:309-22.

47. Sotirova A V., Spasova DI, Galabova DN, Karpenko E, Shulga A. 2008. Rhamnolipidbiosurfactant permeabilizing effects on gram-positive and gram-negative bacterial strains. Curr Microbiol 56:639-644.

48. Ryall B, Carrara M, Zlosnik JEA, Behrends V, Lee X, Wong Z, Lougheed KE, Williams HD.

2014. The mucoid switch in Pseudomonas aeruginosa represses quorum sensing systems and leads to complex changes to stationary phase virulence factor regulation. PLoS One

49. Gifford AH, Willger SD, Dolben EL, Moulton LA, Dorman DB, Bean H, Hill JE, Hampton TH, Immun 84:2995-3006.

50. Liberati NT, Urbach JM, Miyata S, Lee DG, Drenkard E, Wu G, Villanueva J, Wei T, Ausubel transposon insertion mutants. Proc Natl Acad Sci 103:2833-2838.

579 51. May TB, Shinabarger D, Maharaj R, Kato J, Chu L, DeVault JD, Roychoudhury S, Zielinski 
Microbiol Rev 4:191-206.

583 52. Pedersen SS. 1992. Lung infection with alginate-producing, mucoid Pseudomonas aeruginosa in cystic fibrosis. APMIS Suppl 28:1-79.

585 53. Irie Y, Borlee BR, O’Connor JR, Hill PJ, Harwood CS, Wozniak DJ, Parsek MR. 2012. Selfproduced exopolysaccharide is a signal that stimulates biofilm formation in Pseudomonas aeruginosa. Proc Natl Acad Sci U S A 109:20632-6.

588 54. Knutson CA, Jeanes A. 1968. A new modification of the carbazole analysis: Application to 589 heteropolysaccharides. Anal Biochem 24:470-481. 


\section{$591 \quad$ Figure legends.}

592 Figure 1. Exogenous alginate protects S. aureus JE2 from P. aeruginosa PAO1 in co-culture.

593 A) S. aureus JE2 and B) P. aeruginosa PAO1 growth curves over 10 hours in liquid co-culture in

$594 \mathrm{TSB}+/-1 \%$ seaweed-derived or $1 \%$. aeruginosa PAO1-derived alginate. $\mathrm{CFU} / \mathrm{ml}$ were

595 enumerated at indicated time points and $\log _{10}$ transformed. Dotted lines indicate the presence

596 of alginate in the culture. The dashed horizontal line at $2 \log _{10} \mathrm{CFU} / \mathrm{ml}$ indicates the limit of

597 detection. C) Biofilm and D) Planktonic S. aureus JE2 growth after 16 hours of static co-culture

598 in MEM L-gln L-arg +/- $1 \%$ seaweed-derived alginate. CFU/ml were enumerated and $\log _{10}$

599 transformed. Dashed boxes indicate the presence of alginate in the culture. Significance was

600 determined by one-way ANOVA with Dunnett's post-test. a, $p<0.05$ with $S$. aureus JE2 as the

601 reference. $b, p<0.0001$ with $P$. aeruginosa PAO1 + S. aureus JE2 as the reference.

602

603 Figure 2. Culture with mucoid $P$. aeruginosa PAO1 mucA22 delays S. aureus JE2 killing by wild-

604 type P. aeruginosa PA01. Biofilm tri-culture on plastic with S. aureus JE2, P. aeruginosa PAO1, 605 and P. aeruginosa PAO1 mucA22 in MEM L-gln L-arg. CFU/ml were enumerated on MSA and $606 \log _{10}$ transformed. S. aureus JE2 viability in the A) biofilm and B) planktonic fraction after 12

607 hours and S. aureus JE2 viability in the C) biofilm and D) planktonic fraction after 16 hours co-

608 culture. Dashed boxes indicate the presence of a mucoid strain in the culture. Significance was

609 determined by one-way ANOVA with Dunnett's post-test. a, $p<0.05$ with S. aureus JE2 as the

610 reference. $b, \mathrm{p}<0.05$ with $P$. aeruginosa PAO1 + S. aureus JE2 as the reference. 
612 Figure 3. Alginate synthesis is not required for S. aureus protection. S. aureus JE2 was co-

613 cultured in 1/2X MEM L-gln L-arg on plastic with P. aeruginosa PAO1 or P. aeruginosa PAO1

614 algD::FRT +/- 1\% seaweed-derived alginate. S. aureus JE2 viability in the A) biofilm and B)

615 planktonic fractions after 16 hours. P. aeruginosa PAO1 viability in the C) biofilm and D)

616 planktonic fractions after 16 hours. Dashed boxes indicate the presence of alginate in the

617 culture. Significance was determined by one-way ANOVA with Dunnett's post-test. a, $p<0.05$

618 with S. aureus JE2 as the reference. $b, p<0.05$ with $P$. aeruginosa PAO1 + S. aureus JE2 as the

619 reference.

621 Figure 4. Exogenous alginate decreases $P$. aeruginosa PAO1 siderophore production. A-B) $P$.

622 aeruginosa PAO1 was grown on plastic in 1/2X MEM L-gln L-arg +/- 1\% alginate for 16 hours at

$62337^{\circ} \mathrm{C}$ with $5 \% \mathrm{CO}_{2}$. Supernatants were collected from the planktonic fraction. A) Pyoverdine was

624 quantified by measuring RFU of the supernatants at $400 \mathrm{~nm}$ excitation and $460 \mathrm{~nm}$ emission

625 and normalizing to $\mathrm{CFU} / \mathrm{mL}$ of the planktonic fraction. B) Supernatants were diluted $1 / 2 \mathrm{X}$ in DI

626 water or $1 \%$ alginate and incubated statically at $37^{\circ} \mathrm{C}$ with $5 \% \mathrm{CO}_{2}$ for 5 minutes or 24 hours and

627 pyoverdine was quantified by RFU of the supernatants at $400 \mathrm{~nm}$ excitation and $460 \mathrm{~nm}$

628 emission. Significance determined by paired t-test. C-D) P. aeruginosa PAO1 was grown in $25 \mathrm{~mL}$

629 TSB shaking for 8 hours. C) pvdA expression was quantified by q-RT-PCR and ddCt was

630 calculated relative to $P$. aeruginosa PAO1 rpoD expression. D) Pyochelin was quantified by LC-

631 MS/MS. Significance determined by one-way ANOVA with Dunnett's post-test comparison to $P$.

632 aeruginosa PAO1 unless otherwise indicated. For all statistical tests, ${ }^{*} p<0.05, * * p<0.01$, 
Figure 5. Rhamnolipid production is post-transcriptionally altered by exogenous alginate. $P$. aeruginosa PAO1 was grown in TSB in liquid culture for 8 hours +/- 1\% alginate. Supernatants were collected by centrifuging to remove cell debris, and sterile filtering. Cell pellets were snap

638 frozen for expression analyses. A) Representative drop collapse assay image. P. aeruginosa

639 PAO1 supernatants were prepared from mucoid and non-mucoid $P$. aeruginosa PAO1 and

640 serially diluted 1:2 in PBS. Surfactant activity was assessed by placing a 20uL droplet of each

641 supernatant dilution on plastic, placing the droplet at a $90^{\circ}$ angle for 10 seconds and assessing

642 migration (the PBS was supplemented with $0.01 \%$ CV to aid visualization). Surfactant activity

643 was quantified as the reciprocal of the highest dilution at which the drop migrates. B)

644 Rhamnolipid production by $P$. aeruginosa PAO1 quantified by drop collapse and normalized to

$645 \mathrm{CFU} / \mathrm{mL}$ to determine surfactant score. Rhamnolipid quantification by LC-MS/MS for C) total

646 rhamnolipids and D) mono- and di-rhamnolipids. E) rhIA expression was quantified by q-RT-PCR

647 and $\Delta \Delta \mathrm{Ct}$ was calculated relative to $P$. aeruginosa PAO1 rpoD. Significance determined by one-

648 way ANOVA with Dunnett's post-test comparison to P. aeruginosa PAO1. ${ }^{*} \mathrm{p}>0.05,{ }^{*} \mathrm{p}<0.01$,

$649 * * * p<0.001, * * * * p<0.0001$

651 Figure 6. Exogenous alginate downregulates PQS quorum sensing. A) The PQS quorum

652 sensing regulon. PQS synthesis is dependent on the $p q s A B C D$ genes located in the pqs operon.

653 Both HHQ and HQNO have direct antimicrobial properties, while PQS is the ligand for PqsR.

654 When PQS level is high this ligand interacts with PqsR to positively regulate many downstream

655 virulence factors. PQS has direct iron-chelating function and promotes expression of 
656 siderophore-encoding genes. The downstream effects listed here focus on effects relevant to

657 this study and are not exhaustive. B-F) In Panel B is shown is the legend for all graphs in this

658 figure. LC-MS/MS was used to quantify B) HQNO C) HHQ D) PQS E) PCA and F) pyocyanin

659 produced by $P$. aeruginosa.

660

661 Figure 7. Alginate alters the expression of $\boldsymbol{P}$. aeruginosa genes essential for S. aureus killing.

662 A-D) Raw Nanostring counts were normalized to positive controls and three housekeeping

663 genes $(r p o D, p p i D, f b p)$ and $\log _{2}$ transformed. Three biological replicates per condition.

664 Significantly differentially expressed genes were determined by unpaired $t$-test followed by the

665 two-stage linear step-up procedure of Benjamini, Krieger, and Yekutieli (with $q 1 \%$ for false

666 discovery), and are marked by blue squares and labeled with the gene name. Genes

667 significantly differentially expressed by mucoid $P$. aeruginosa in a previous study (32) but not by

$668 P$. aeruginosa in the presence of exogenous alginate are marked by red triangles. A) $P$.

669 aeruginosa PAO1 and B) P. aeruginosa PA14 sub-cultured into TSB or TSB $+1 \%$ alginate during

670 mid-log growth phase for 45 minutes. For clarity, only significantly downregulated genes are

671 labeled in B). P. aeruginosa PA14 C) mono-cultured and D) co-cultured with S. aureus JE2 in TSB

$672+0.25 \%$ alginate for 8 hours. E-F) S. aureus JE2 survival in the E) biofilm and F) planktonic

673 fractions after 16 hour co-culture with $P$. aeruginosa PA14 mutants for genes downregulated in

674 one or more Nanostring experiment. Significance determined by one-way ANOVA with

675 Dunnett's post-test comparison to S. aureus in monoculture. ${ }^{*} p<0.05,{ }^{* *} p<0.01,{ }^{* * *} p<0.001$,

$676 * * * * p<0.0001$. G-H) S. aureus JE2 survival in the G) biofilm and H) planktonic fractions after 16

677 hour co-culture with P. aeruginosa PA14 motility mutants. Significance determined by one-way 
678 ANOVA with Dunnett's post-test. $a, p<0.05$ with S. aureus JE2 as the reference. $b, p<0.05$ with

679 P. aeruginosa PAO1 + S. aureus JE2 as the reference.

680

681 Figure 8. Mucoid clinical isolates have varying effects on S. aureus in coculture. A) S. aureus

682 JE2 growth after 16 and 24 hours of co-culture in flasks shaking at $225 \mathrm{rpm}$ in TSB with $P$.

683 aeruginosa clinical isolates. $\mathrm{Nm}=$ nonmucoid. $\mathrm{B}-\mathrm{C}) \log _{2}$ transformation of Nanostring counts

684 normalized to positive controls and three housekeeping genes $(r p o D, p p i D, f b p)$ for the

685 indicated transcripts for clinical isolate $P$. aeruginosa CFBRPA32, mucoid laboratory strain $P$.

686 aeruginosa PAO1 mucA22, and wild-type laboratory strain P. aeruginosa PAO1 after 24 hours

687 culture in flasks shaking at $225 \mathrm{rpm}$ in TSB. Two biological replicates per strain. Gene expression

688 was analyzed by two-way ANOVA followed by Tukey's multiple comparisons. B) Heatmap and

689 dendrogram of all genes significantly differentially regulated between any two strains.

690 Expression values displayed as within-row Z-scores. Yellow indicates mucoid strains, and gray

691 indicates non-mucoid strains. C) All genes significantly differentially regulated between $P$.

692 aeruginosa CFBRPA32 and P. aeruginosa PAO1 mucA22. ${ }^{*} p<0.05, * * p<0.01, * * * p<0.001$,

$693 * * * * p<0.0001$

694

695 Figure S1. Exogenous alginate protects S. aureus JE2 from P. aeruginosa PAO1 in co-culture.

696 A) S. aureus JE2 and B) P. aeruginosa PAO1 growth after 8 hours of liquid co-culture in TSB +/-

697 1\% seaweed-derived or P. aeruginosa PAO1-derived alginate. CFU/ml were enumerated and

$698 \log _{10}$ transformed. Dashed boxes indicate the presence of alginate in the culture. Significance

699 was determined by one-way ANOVA with Dunnett's post-test. a, $p<0.0001$ with S. aureus JE2 as 
700 the reference. $b, p<0.0001$ for panel $\mathbf{A}$ and $p<0.05$ for panel $\mathbf{B}$ with $P$. aeruginosa PAO1 $+S$.

701 aureus JE2 as the reference.

702

703 Figure S2. Exogenous alginate protects S. aureus JE2 from P. aeruginosa in co-culture.

704 Liquid co-cultures of S. aureus JE2 and P. aeruginosa in TSB +/- seaweed-derived alginate added

705 at $0.25 \%$ or $2 \%$. CFU/ml were $\log _{10}$ transformed. A) S. aureus JE2 and B) P. aeruginosa PAO1

706 growth curves over 8 hours in monoculture or the indicated cocultures. CFU/ml were

707 enumerated at indicated time points. C) S. aureus JE2 and D) P. aeruginosa PAO1 growth after 8

708 hours. E) S. aureus JE2 and F) P. aeruginosa PA14 growth curves over 8 hours. CFU/ml were

709 enumerated at indicated time points. G) S. aureus JE2 and H) P. aeruginosa PA14 growth after 8

710 hours. Dotted lines/dashed boxes indicate the presence of alginate in the culture. Significance

711 was determined by one-way ANOVA with Dunnett's post-test. a, $\mathrm{p}<0.05$ with S. aureus JE2 as

712 the reference. $b, p<0.001$ with $P$. aeruginosa + S. aureus JE2 as the reference.

714 Figure S3. Exogenous alginate protects S. aureus JE2 from P. aeruginosa in co-culture.

715 A-D) Liquid co-cultures of S. aureus JE2 and P. aeruginosa PAO1 in TSB +/- seaweed-derived

716 alginate added at $0.25 \%$ or $2 \%$. P. aeruginosa initial density was $10 \mathrm{X}$ higher than S. aureus

717 density based on $\mathrm{OD}_{600}$. $\mathrm{CFU} / \mathrm{ml}$ were $\log _{10}$ transformed. A) S. aureus JE2 and B) P. aeruginosa

718 PAO1 growth curves over 8 hours. CFU/ml were enumerated at indicated time points. The

719 dashed line at 2 indicates the limit of detection. C) S. aureus JE2 and D) P. aeruginosa PAO1

720 growth after 8 hours. E-F) Biofilm co-cultures of $P$. aeruginosa PAO1 and S. aureus JE2 for 16 hrs

721 in MEM L-gln L-arg +/- 1\% seaweed-derived alginate. CFU/ml were enumerated and $\log _{10}$ 
722 transformed. P. aeruginosa PAO1 E) biofilm and F) planktonic growth. G-J) Biofilm co-cultures

723 of $P$. aeruginosa PA14 and S. aureus JE2 for 16 hours in MEM L-gln L-arg +/- 1\% seaweed-

724 derived alginate. CFU/ml were enumerated and $\log _{10}$ transformed. S. aureus JE2 G) biofilm and

725 H) planktonic and P. aeruginosa PA14 I) biofilm and J) planktonic growth. Dotted lines/dashed

726 boxes indicate the presence of alginate in the culture. Significance was determined by one-way

727 ANOVA with Dunnett's post-test. a, $p<0.05$ with S. aureus JE2 as the reference. $b, p<0.05$ with

728 P. aeruginosa + S. aureus JE2 as the reference.

730 Figure S4. Exogenous alginate protects $S$. aureus JE2 from $P$. aeruginosa in minimal medium

731 liquid co-culture. A) S. aureus JE2 and B) P. aeruginosa PAO1 and P. aeruginosa PA14 growth

732 after 20 hours co-culture in 1/2X MEM L-gln L-arg +/- 1\% alginate. CFU/ml were enumerated

733 and $\log _{10}$ transformed. Significance determined by unpaired t-test. ${ }^{*} p<.05, * * p<.01, * * * p<.001$.

735 Figure S5. Culture with mucoid P. aeruginosa PAO1 mucA22 delays S. aureus JE2 killing by

736 wild-type $P$. aeruginosa PAO1. Biofilm tri-culture on plastic with S. aureus JE2, P. aeruginosa

737 PAO1, and P. aeruginosa PAO1 mucA22 in MEM L-gln L-arg. CFU/ml were enumerated on MSA

738 and $\log _{10}$ transformed. Growth curve of S. aureus JE2 viability in the A) biofilm and B)

739 planktonic fraction. Dashed lines indicate the presence of a mucoid strain in the culture. $P$.

740 aeruginosa PAO1 growth in the C) biofilm and D) planktonic fraction after 12 hours and $P$.

741 aeruginosa PAO1 growth in the E) biofilm and F) planktonic fraction after 16 hours co-culture.

742 Dashed boxes indicate the presence of a mucoid strain in the culture. Significance was 
743 determined by one-way ANOVA with Dunnett's post-test. a, $p<0.05$ with S. aureus JE2 as the

744 reference. $b, \mathrm{p}<0.05$ with $P$. aeruginosa PAO1 + S. aureus JE2 as the reference.

746 Figure S6. P. aeruginosa PA14 gene expression changes in response to S. aureus JE2 and

747 alginate. Transcriptional profiling of $P$. aeruginosa by Nanostring revealed that the presence of

748 S. aureus has a stronger impact on overall $P$. aeruginosa gene expression than exogenous

749 alginate. P. aeruginosa PA14 was cultured for 8 hours in TSB +/- $0.25 \%$ alginate and +/- S.

750 aureus JE2. Nanostring counts were normalized to positive controls and three housekeeping

751 genes $(r p o D, p p i D, f b p)$ and $\log _{2}$ transformed. P. aeruginosa PA14 gene expression was driven

752 more by exposure to S. aureus JE2 than exposure to exogenous alginate. A) Clustering by

753 Euclidean distance of the average of three biological replicates. Image was generated in R with

754 the Heatmap.2 function. B) Principal component analysis of all biological replicates. Samples

755 separate more strongly on the presence of S. aureus (PCA 1) than on the presence of exogenous

756 alginate (PCA 2).

757

758 Figure S7. P. aeruginosa PA14 mutants do not have growth defects. Growth of $P$. aeruginosa

759 PA14 mutants after 16 hours co-culture on plastic with S. aureus JE2 in MEM L-gln L-arg. A)

760 Biofilm and B) planktonic fractions for initial mutant screen. C) Biofilm and D) planktonic

761 fractions for flagellar mutant screen. Significance determined by one-way ANOVA with

762 Dunnett's post-test comparison to wild-type P. aeruginosa PA14. ${ }^{*} \mathrm{p}<0.05$. 


\section{$764 \quad$ Figure S8. Gene expression of $\boldsymbol{P}$. aeruginosa clinical isolates and nonmucoid revertants.}

765 Heatmap and dendrogram of a subset of genes in the Nanostring codeset from P. aeruginosa

766 clinical isolates cultured in lysogeny broth (LB) for 8-11 hours (Gifford AH, Willger SD, Dolben

767 EL, Moulton LA, Dorman DB, Bean H, Hill JE, Hampton TH, Ashare A, Hogan DA. 2016. Infect

768 Immun 84:2995-3006.). $\log _{2}$ transformation of Nanostring counts were normalized to positive

769 controls from a single biological replicate. Expression values are displayed as within-row Z-

770 scores. Yellow indicates mucoid strains, and gray indicates non-mucoid strains. Image

771 generated in R with Heatmap.2 function. This dataset contains expression data for the isogenic

772 nonmucoid revertants of $P$. aeruginosa FRD1 and $P$. aeruginosa $\mathrm{Cl} 2224$. Here we display a direct

773 comparison of gene expression for these six clinical isolates and two laboratory P. aeruginosa

774 strains of a subset of genes in the Nanostring codeset. Interestingly, isolates cluster primarily on

775 mucoidy, and the mucoid isolates which were better able to kill S. aureus (P. aeruginosa FRD1

776 and $P$. aeruginosa $\mathrm{Cl} 228$ ) clustered together within those groups. Mucoid $P$. aeruginosa FRD1

777 decreases some genes as expected given its mucoid phenotype, including $f l g G, f l g D, f l g K$, and

778 flic. However, $r h l A, p h z$, and $p v d A$ expression remain high; all of these genes are known to

779 contribute to the ability of $P$. aeruginosa to kill S. aureus in co-culture (Limoli DH, Hoffman LR.

780 2019. Thorax thoraxjnl-2018-212616.). P. aeruginosa $\mathrm{Cl} 228$ has a similar gene expression

781 pattern to $P$. aeruginosa FRD1, but pvdA expression is even higher in this strain. $P$. aeruginosa

$782 \mathrm{Cl} 2224$, which is similar to P. aeruginosa CFBRPA32 in the ability to kill S. aureus (that is, both

783 strains show limited ability to kill S. aureus) downregulates a large number of genes coding for

784 known virulence factors, including those genes required for the production of rhamnolipids

$785(r h l A, r h l l)$, phenazines (phzA, phzC), quorum sensing factors ( $p q s H, p q s A)$, and flagella $(f l g D$, 
$786 f(g G, f l i C)$. It is not immediately clear based on these data why this isolate is able to kill S. aureus

787 at the late time points. P. aeruginosa CFRL8, which is the poorest of these four strains at killing

788 S. aureus, highly expresses $p c h C$ and flic, but has lower pqsA expression relative to the other

789 strains. To determine whether we could broadly correlate the expression of any particular

790 genes with changes in virulence towards S. aureus, we plotted the expression of each gene

791 against $S$. aureus survival in co-culture at both 16 and 24 hours for each $P$. aeruginosa strain

792 where we have Nanostring data and performed a linear regression (data not shown). We

793 observed by this method that no individual gene's expression is significantly correlated with S.

794 aureus survival. Taken together, these data suggest that across clinical mucoid strains, the

795 relative production of a variety of transcripts such as those encoding pyoverdine, rhamnolipids,

796 flagella, and phenazines determines whether isolates of $P$. aeruginosa effectively outcompete $S$.

797 aureus, but the specific mechanisms vary on a strain-by-strain basis. 

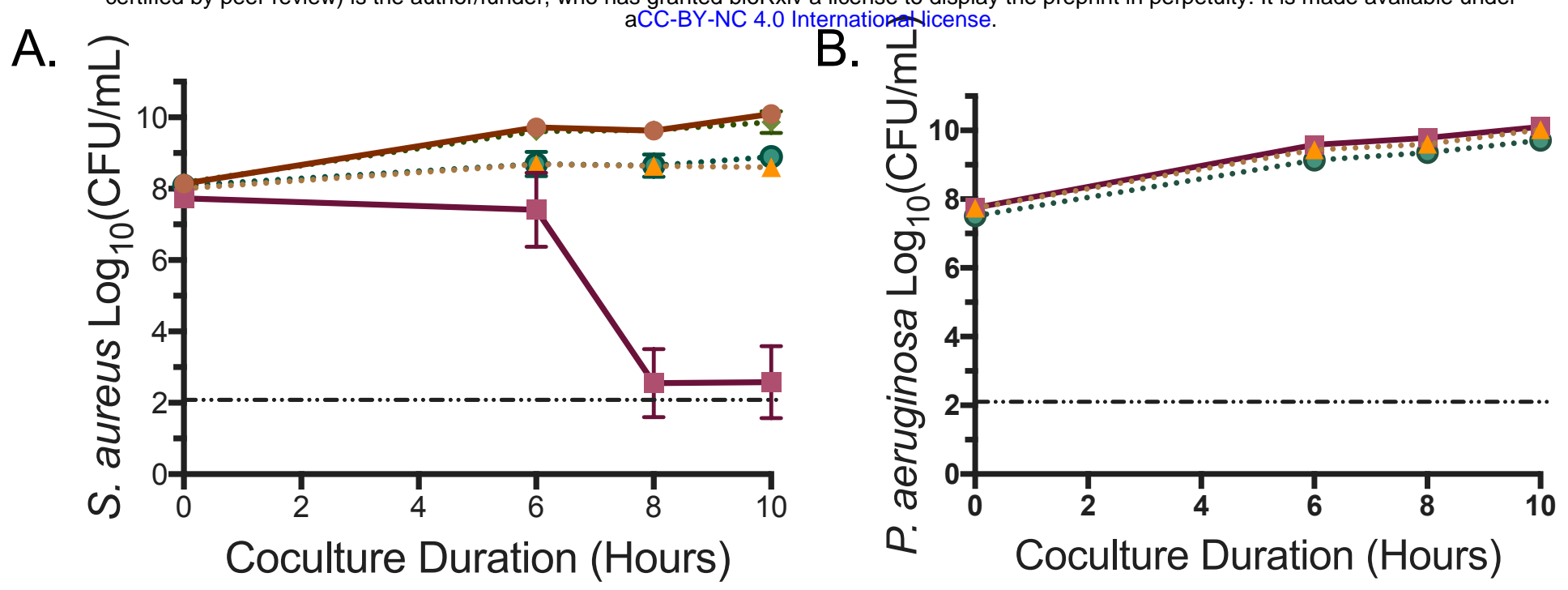

$$
\begin{aligned}
& \begin{array}{l}
\text { - Sa JE2 or Pa PAO1 } \cdot \text { - } \cdot \text { Sa JE2 + } 1 \% \text { Pa JE2 + Pa PAO1 + 1\% Pa PAO1 alginate } \\
\text { - Sa JE2 + Pa PAO1 }
\end{array} \\
& \cdots \text { Sa JE2 + Pa PAO1 + 1\% seaweed alginate }
\end{aligned}
$$

C

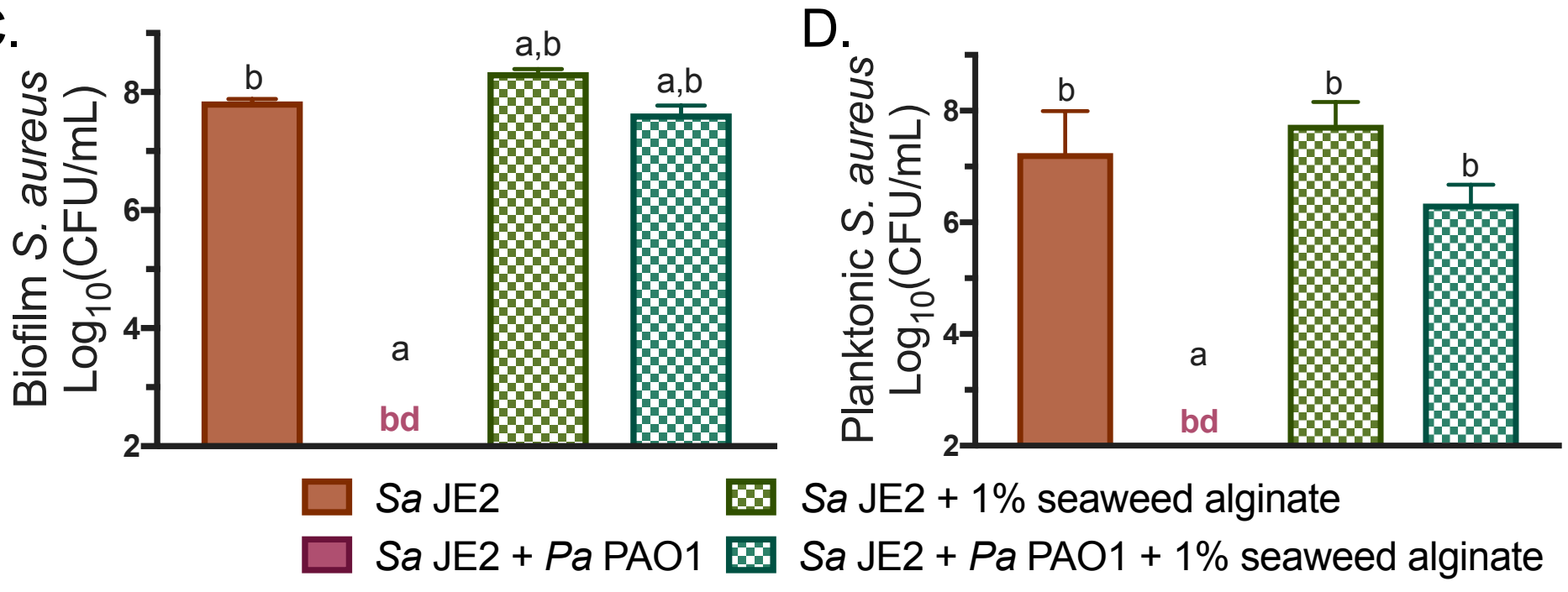


Figure 1. Exogenous alginate protects $S$. aureus JE2 from $P$. aeruginosa PAO1 in co-culture.

A) S. aureus JE2 and B) P. aeruginosa PAO1 growth curves over 10 hours in liquid co-culture in

TSB +/- $1 \%$ seaweed-derived or $1 \%$ P. aeruginosa PAO1-derived alginate. CFU $/ \mathrm{ml}$ were

enumerated at indicated time points and $\log _{10}$ transformed. Dotted lines indicate the presence of alginate in the culture. The dashed horizontal line at $2 \log _{10} \mathrm{CFU} / \mathrm{ml}$ indicates the limit of detection. C) Biofilm and D) Planktonic S. aureus JE2 growth after 16 hours of static co-culture in MEM L-gln L-arg +/- 1\% seaweed-derived alginate. CFU/ml were enumerated and $\log _{10}$ transformed. Dashed boxes indicate the presence of alginate in the culture. Significance was determined by one-way ANOVA with Dunnett's post-test. a, $p<0.05$ with $S$. aureus JE2 as the reference. $b, p<0.0001$ with $P$. aeruginosa PAO1 $+S$. aureus JE2 as the reference. 
A.

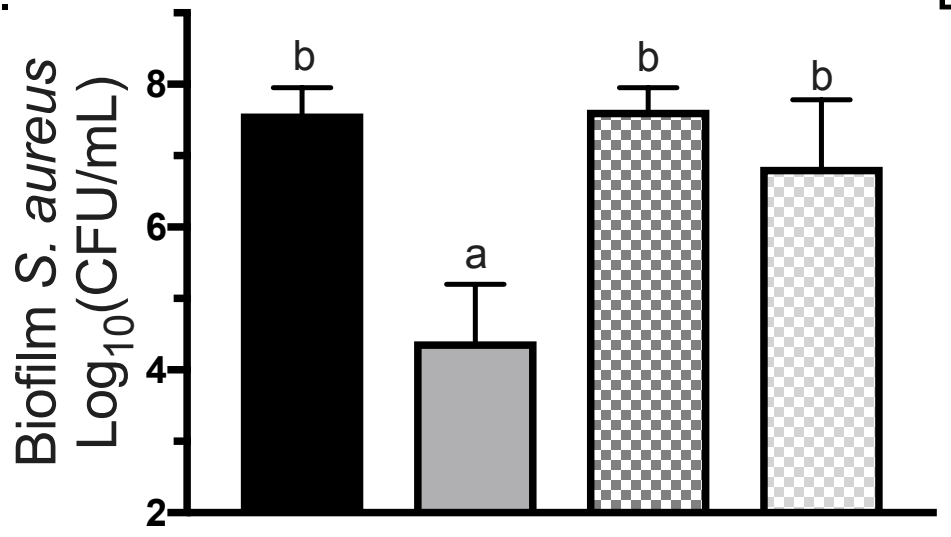

C.

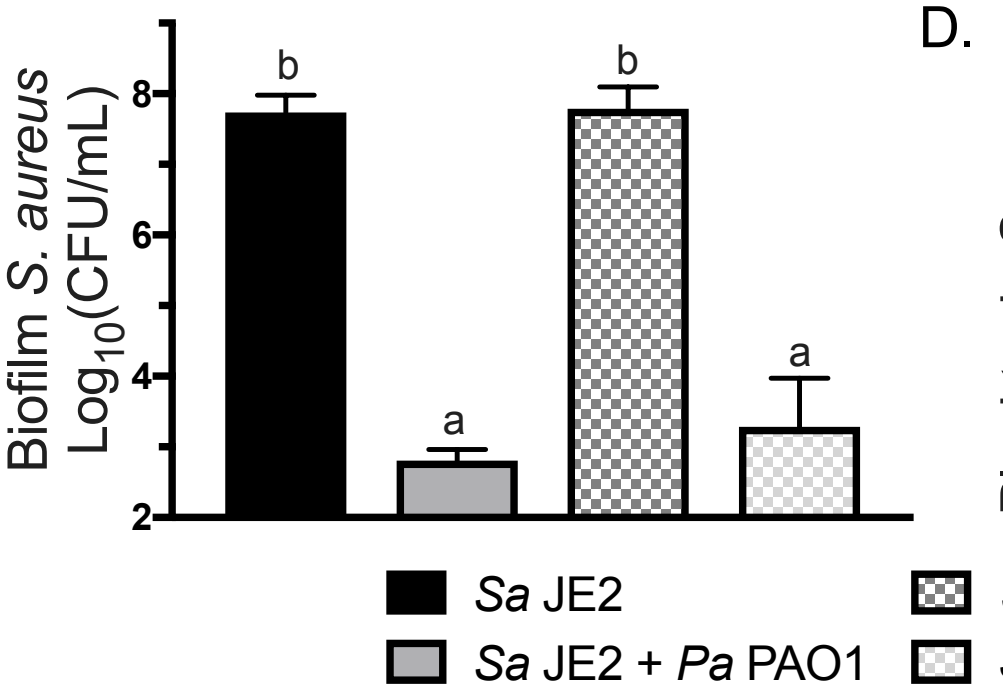

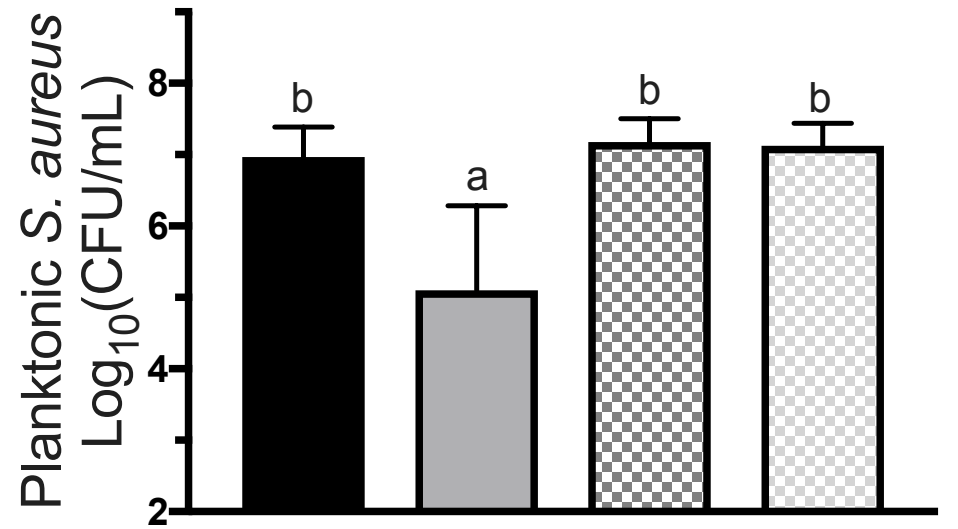

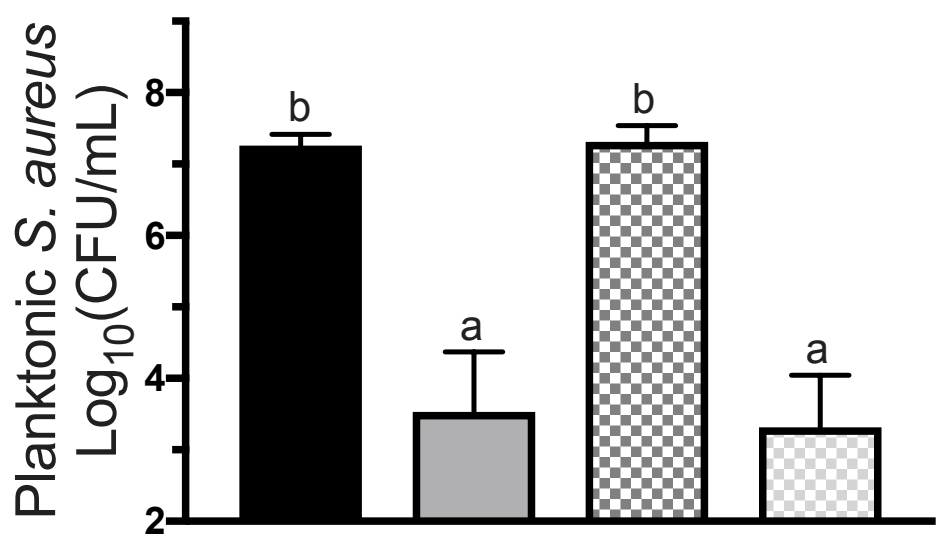

Sa JE2 + Pa PAO1 mucA22

Sa JE2 + Pa PAO1 + Pa PAO1 mucA22

Figure 2. Culture with mucoid P. aeruginosa PAO1 mucA22 delays S. aureus JE2 killing by

wild-type $P$. aeruginosa PAO1. Biofilm tri-culture on plastic with S. aureus JE2, $P$. aeruginosa PAO1, and $P$. aeruginosa PAO1 mucA22 in MEM L-gln L-arg. CFU/ml were enumerated on MSA and $\log _{10}$ transformed. S. aureus JE2 viability in the A) biofilm and B) planktonic fraction after 12 hours and S. aureus JE2 viability in the C) biofilm and D) planktonic fraction after 16 hours co-culture. Dashed boxes indicate the presence of a mucoid strain in the culture. Significance was determined by one-way ANOVA with Dunnett's post-test. a, $p<0.05$ with S. aureus JE2 as the reference. $b, p<0.05$ with $P$. aeruginosa PAO1 $+S$. aureus JE2 as the reference. 
bioRxiv preprint doi: https://doi.org/10.1101/748459; this version posted August 28, 2019. The copyright holder for this preprint (which was not

A. certified by peer review) is the author/funder, who has granted bioRxiv a license to display the preprint in perpetuity. It is made available under
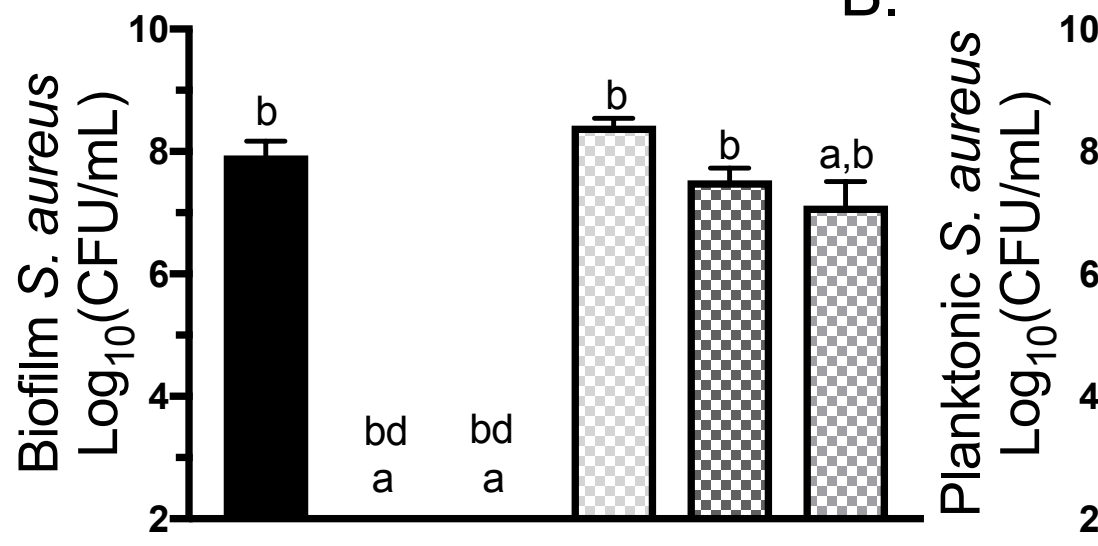

10

Sa JE2 $+\quad+\quad+\quad+\quad+\quad+$

Pa PAO1 - +

Pa PAO1 algD- - $\quad-\quad+\quad+\quad-\quad-\quad+$

$1 \%$ alginate $-\quad-\quad+\quad+\quad+$

C.

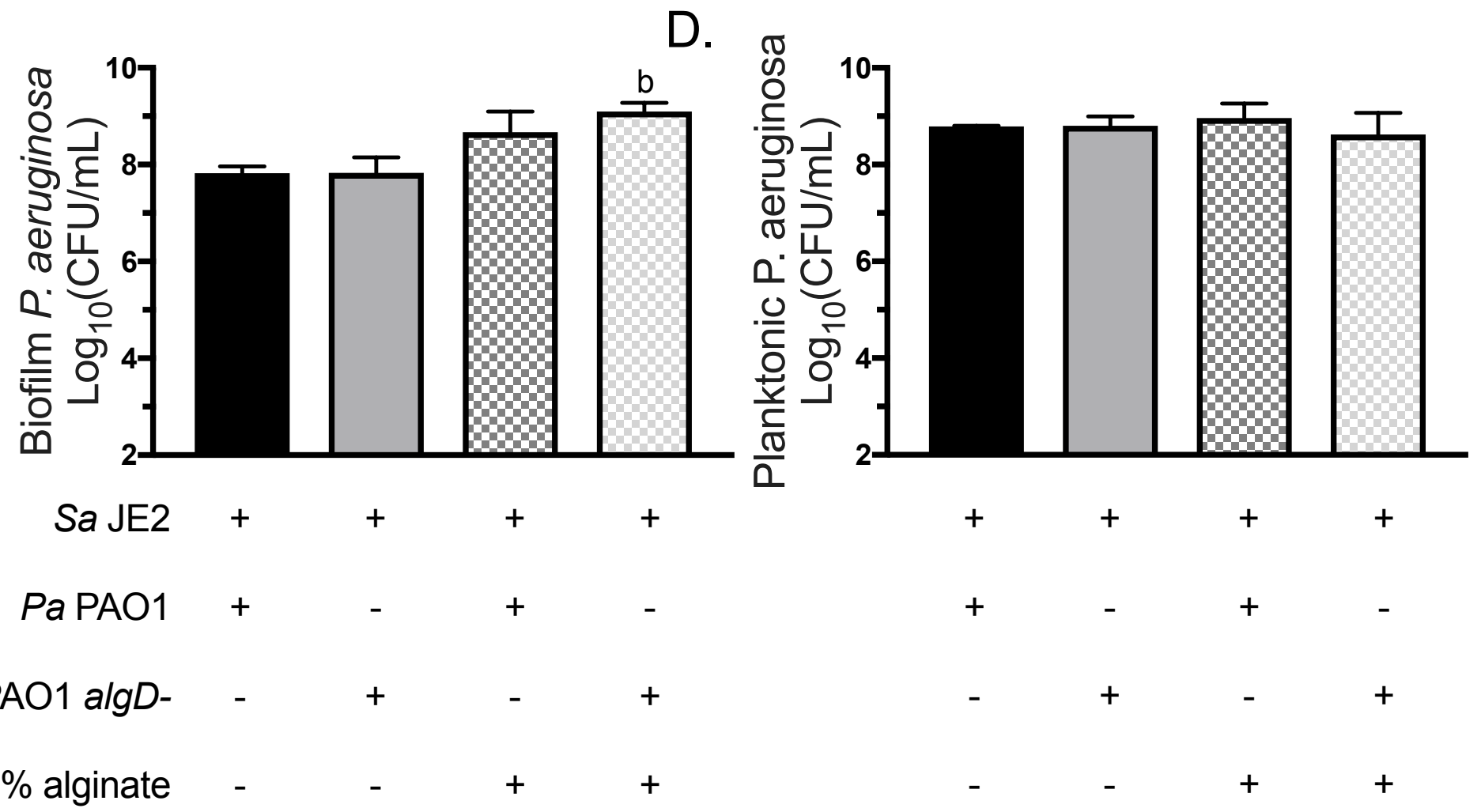


bioRxiv preprint doi: https://doi.org/10.1101/748459; this version posted August 28, 2019. The copyright holder for this preprint (which was not certified by peer review) is the author/funder, who has granted bioRxiv a license to display the preprint in perpetuity. It is made available under aCC-BY-NC 4.0 International license.

Figure 3. Alginate synthesis is not required for S. aureus protection. S. aureus JE2 was cocultured in 1/2X MEM L-gln L-arg on plastic with P. aeruginosa PAO1 or P. aeruginosa PAO1 algD::FRT +/- 1\% seaweed-derived alginate. S. aureus JE2 viability in the A) biofilm and B) planktonic fractions after 16 hours. P. aeruginosa PAO1 viability in the C) biofilm and D) planktonic fractions after 16 hours. Dashed boxes indicate the presence of alginate in the culture. Significance was determined by one-way ANOVA with Punnet's post-test. $a, p<0.05$ with $S$. aureus JE2 as the reference. $b, \mathrm{p}<0.05$ with $P$. aeruginosa $\mathrm{PAO} 1+S$. aureus JE2 as the reference. 
A.

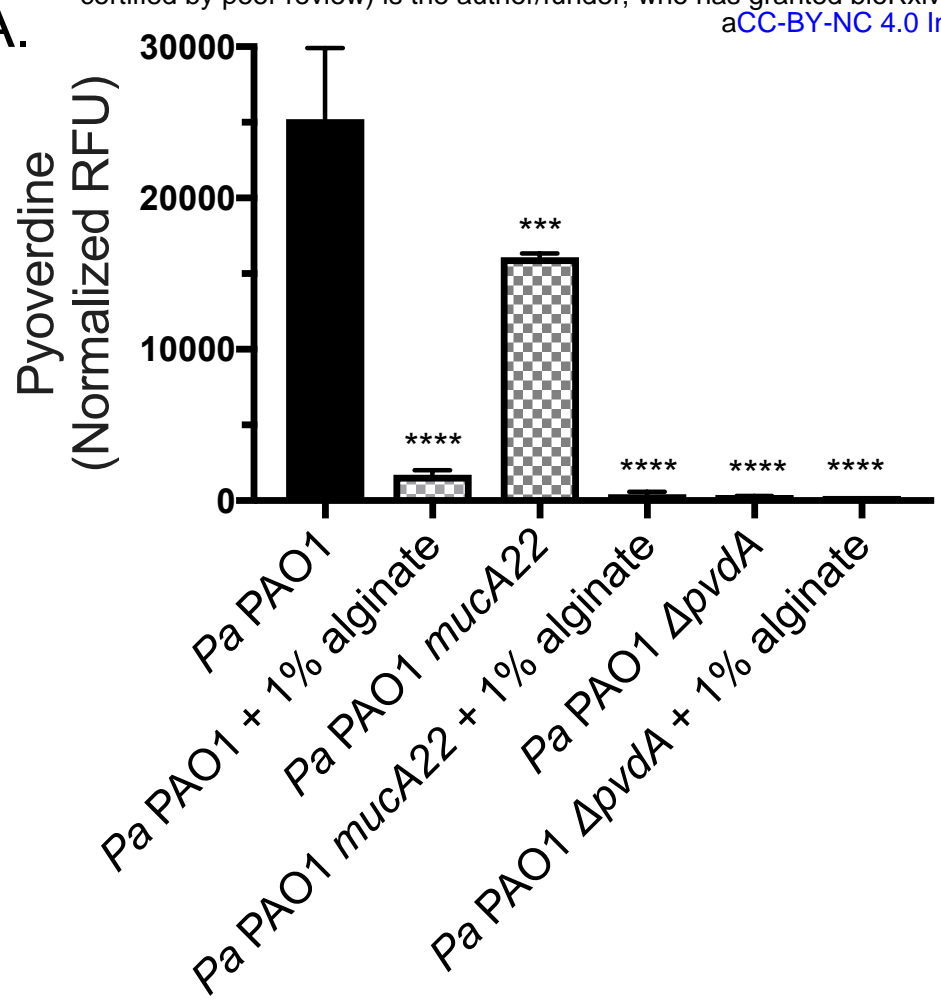

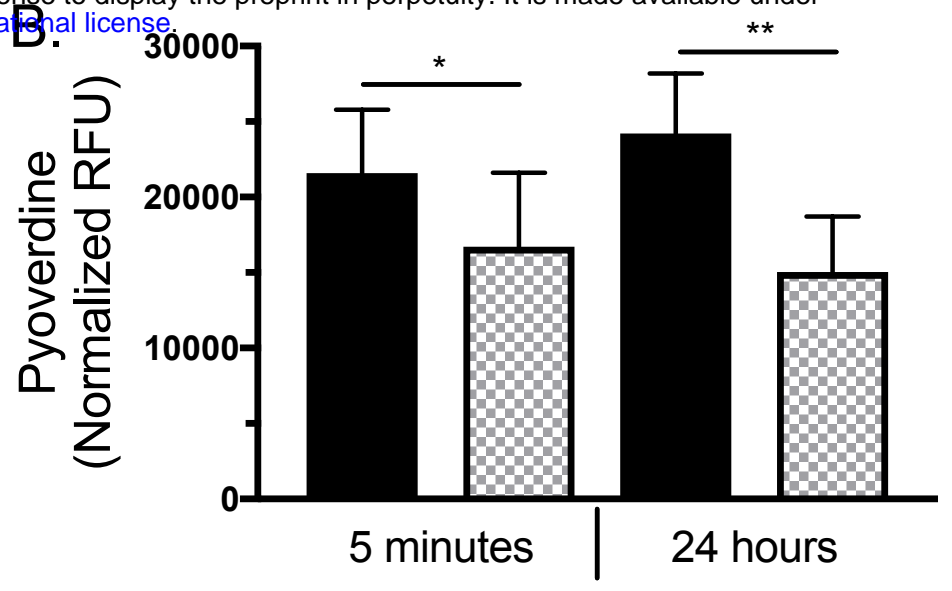

- Pa PAO1 + DI water

$\square \mathrm{PaPAO} 1+1 \%$ alginate
C.

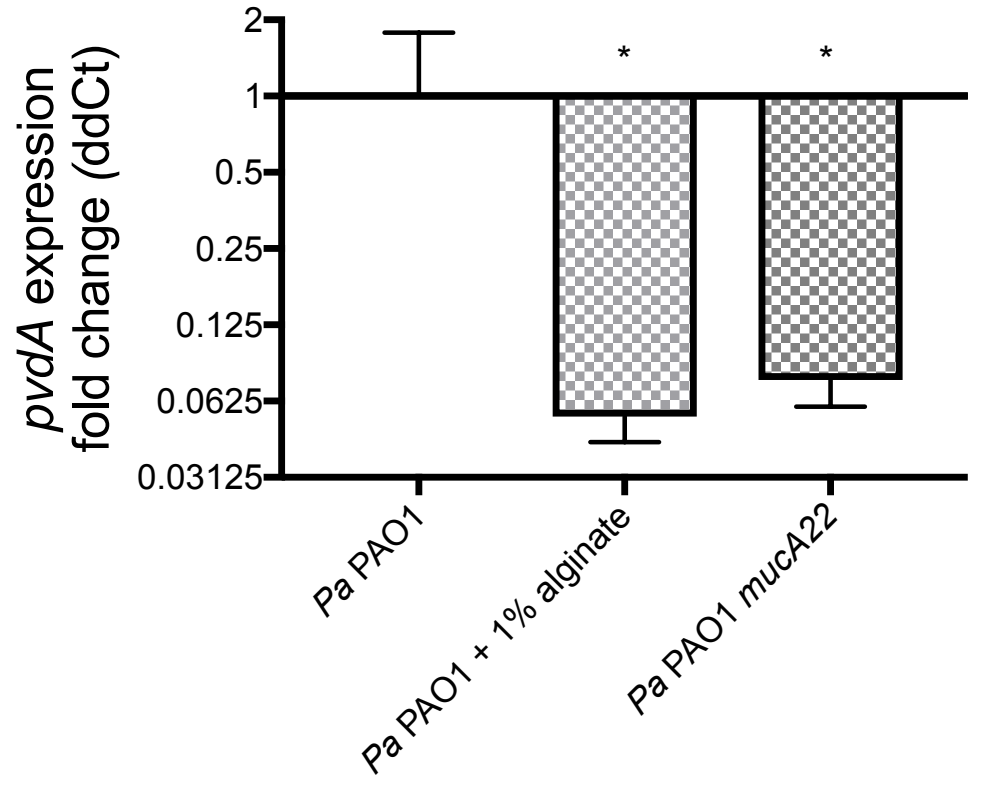

D.

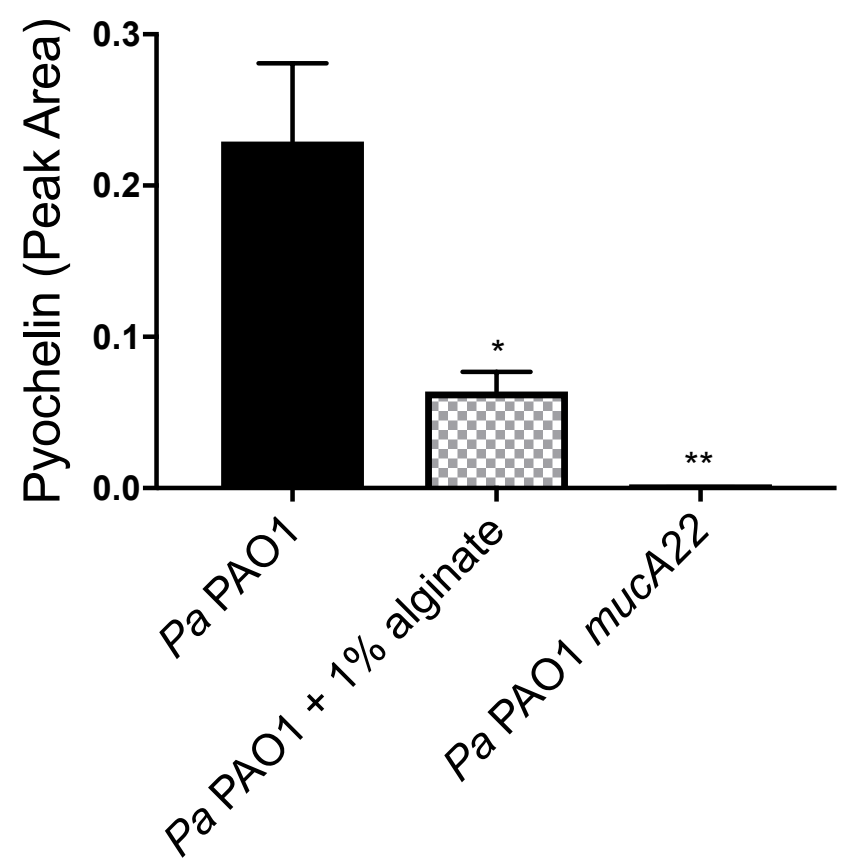


Figure 4. Exogenous alginate decreases $P$. aeruginosa PAO1 siderophore production. A-B) $P$.

aeruginosa PAO1 was grown on plastic in 1/2X MEM L-gln L-arg +/- 1\% alginate for 16 hours at $37^{\circ} \mathrm{C}$ with $5 \% \mathrm{CO}_{2}$. Supernatants were collected from the planktonic fraction. A) Pyoverdine was quantified by measuring RFU of the supernatants at $400 \mathrm{~nm}$ excitation and $460 \mathrm{~nm}$ emission and normalizing to $\mathrm{CFU} / \mathrm{mL}$ of the planktonic fraction. B) Supernatants were diluted $1 / 2 \mathrm{X}$ in DI water or $1 \%$ alginate and incubated statically at $37^{\circ} \mathrm{C}$ with $5 \% \mathrm{CO}_{2}$ for 5 minutes or 24 hours and pyoverdine was quantified by RFU of the supernatants at $400 \mathrm{~nm}$ excitation and $460 \mathrm{~nm}$ emission. Significance determined by paired t-test. C-D) P. aeruginosa PAO1 was grown in $25 \mathrm{~mL}$ TSB shaking for 8 hours. C) $p v d A$ expression was quantified by q-RT-PCR and ddCt was calculated relative to $P$. aeruginosa PAO1 rpoD expression. D) Pyochelin was quantified by LC-MS/MS. Significance determined by oneway ANOVA with Dunnett's post-test comparison to $P$. aeruginosa PAO1 unless otherwise indicated. For all statistical tests, $* p<0.05, * * p<0.01, * * * p<0.001, * * * * p<0.0001$. 
bioRxiv preprint doi: https://doi.org/10.1101/748459; this version posted Auqust 28, 2019. The copvright holder for this preprint (which was not certified by peer review) is the author/funder, who has granted bioRxiv a license to display the preprint in perpetuity. It is made available under A. aCC-BY-NC 4.0 Inte Bational liqense.
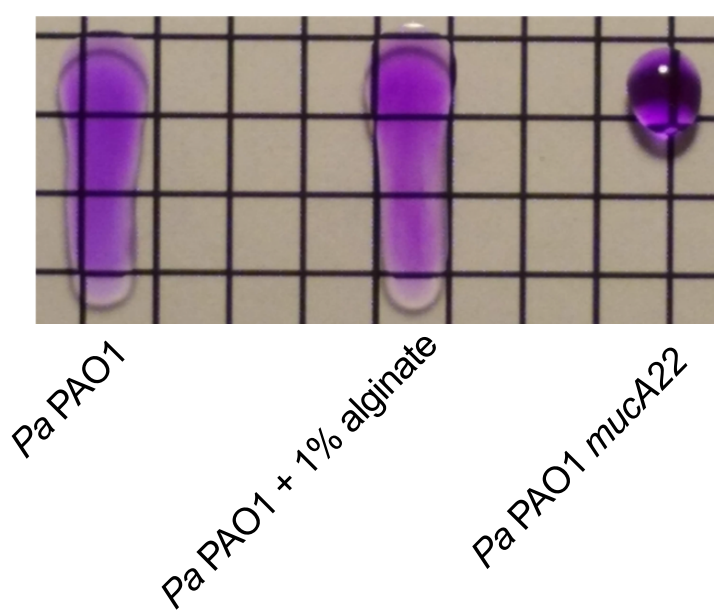

C.
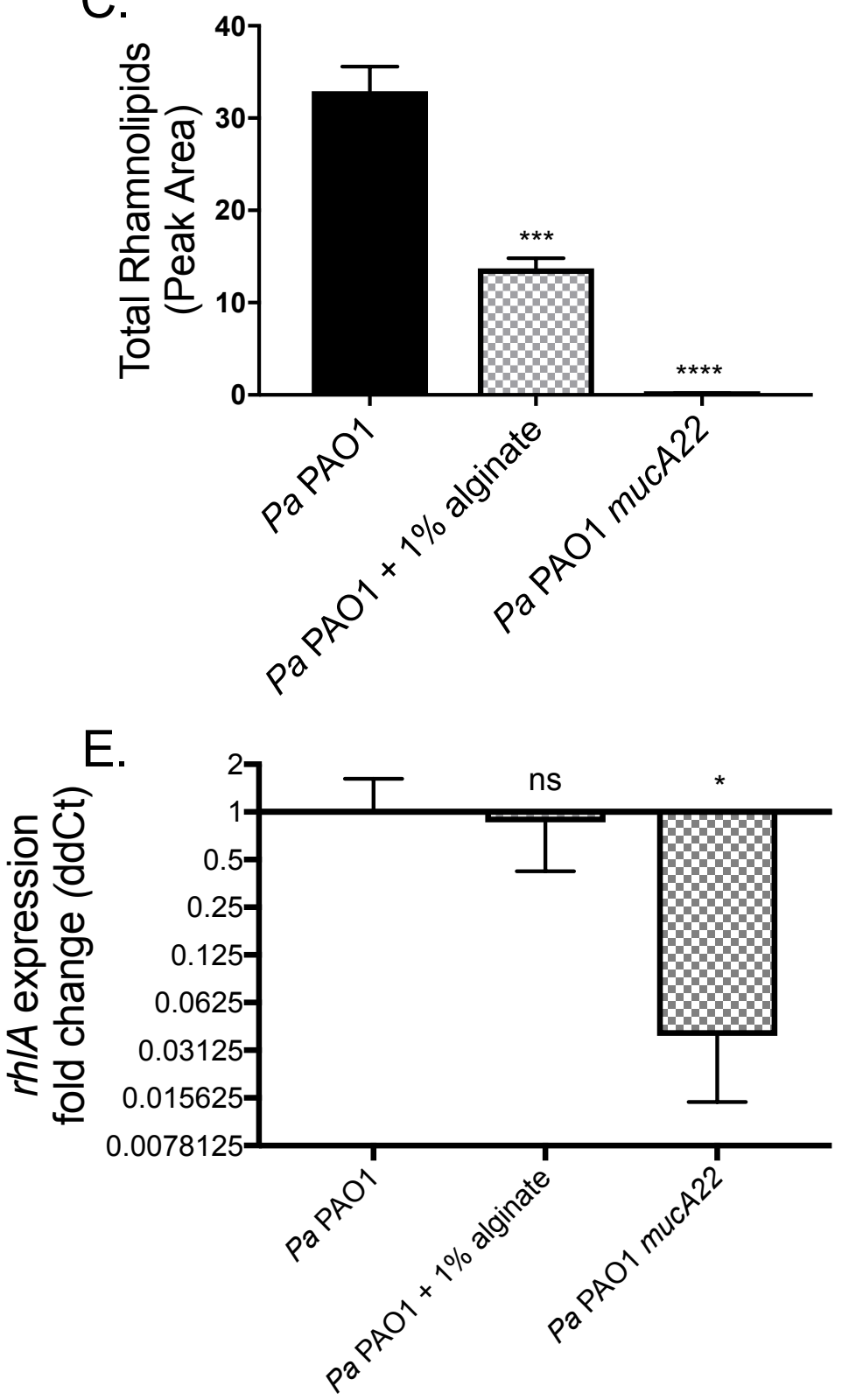

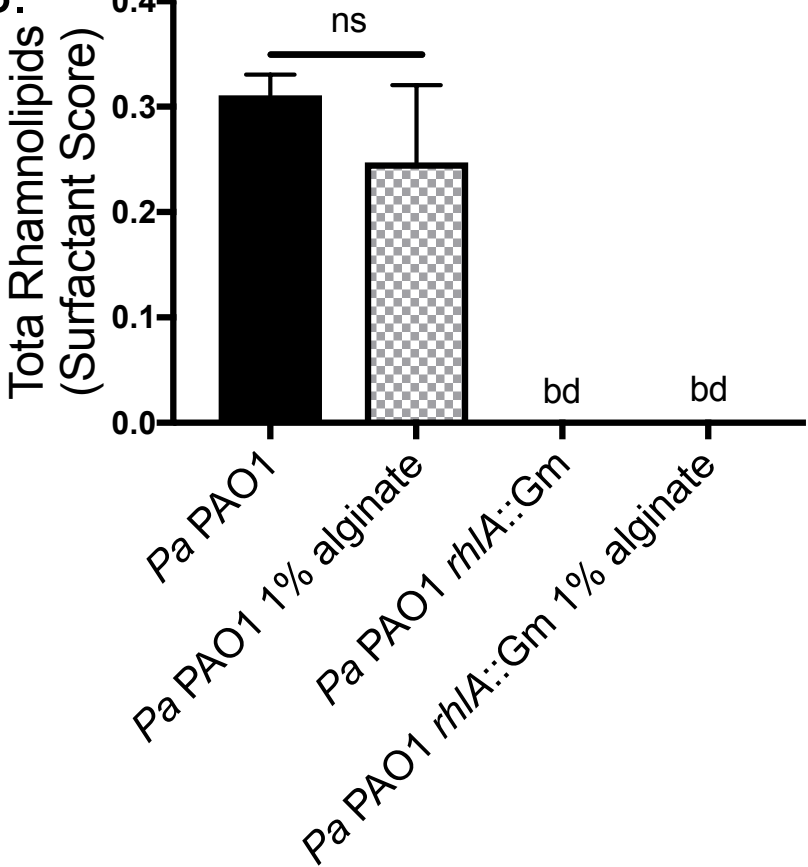

D.

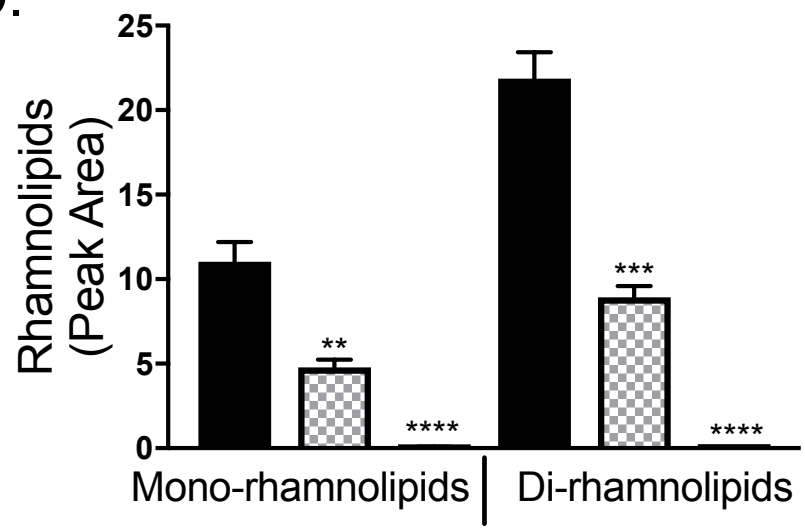

Pa PAO1

PaPAO1 $+1 \%$ alginate

B PaPAO1 mucA22 
Figure 5. Rhamnolipid production is post-transcriptionally altered by exogenous alginate.

P. aeruginosa PAO1 was grown in TSB in liquid culture for 8 hours $+/-1 \%$ alginate.

Supernatants were collected by centrifuging to remove cell debris, and sterile filtering. Cell

pellets were snap frozen for expression analyses. A) Representative drop collapse assay

image. P. aeruginosa PAO1 supernatants were prepared from mucoid and non-mucoid $P$.

aeruginosa PAO1 and serially diluted 1:2 in PBS. Surfactant activity was assessed by placing

a $20 \mathrm{uL}$ droplet of each supernatant dilution on plastic, placing the droplet at a $90^{\circ}$ angle for

10 seconds and assessing migration (the PBS was supplemented with $0.01 \%$ CV to aid

visualization). Surfactant activity was quantified as the reciprocal of the highest dilution at

which the drop migrates. B) Rhamnolipid production by P. aeruginosa PAO1 quantified by

drop collapse and normalized to $\mathrm{CFU} / \mathrm{mL}$ to determine surfactant score. Rhamnolipid

quantification by LC-MS/MS for C) total rhamnolipids and D) mono- and di-rhamnolipids. E)

rhlA expression was quantified by $\mathrm{q}-\mathrm{RT}-\mathrm{PCR}$ and $\Delta \Delta \mathrm{Ct}$ was calculated relative to $P$.

aeruginosa PAO1 rpoD. Significance determined by one-way ANOVA with Dunnett's post-

test comparison to P. aeruginosa PAO1. ${ }^{*} p>0.05,{ }^{* *} p<0.01,{ }^{* * *} p<0.001, * * * * p<0.0001$. 
bioRxiv preprint doi: https://doi.org/10.1101/748459; this version posted August 28, 2019. The copyright holder for this preprint (which was not

A. certified by peer review) is the author/funder, who has granted bioRxiv a license to display the preprint in perpetuity. It is made available under A. Anthranilic acid aCC-BY-NC 4 International license.
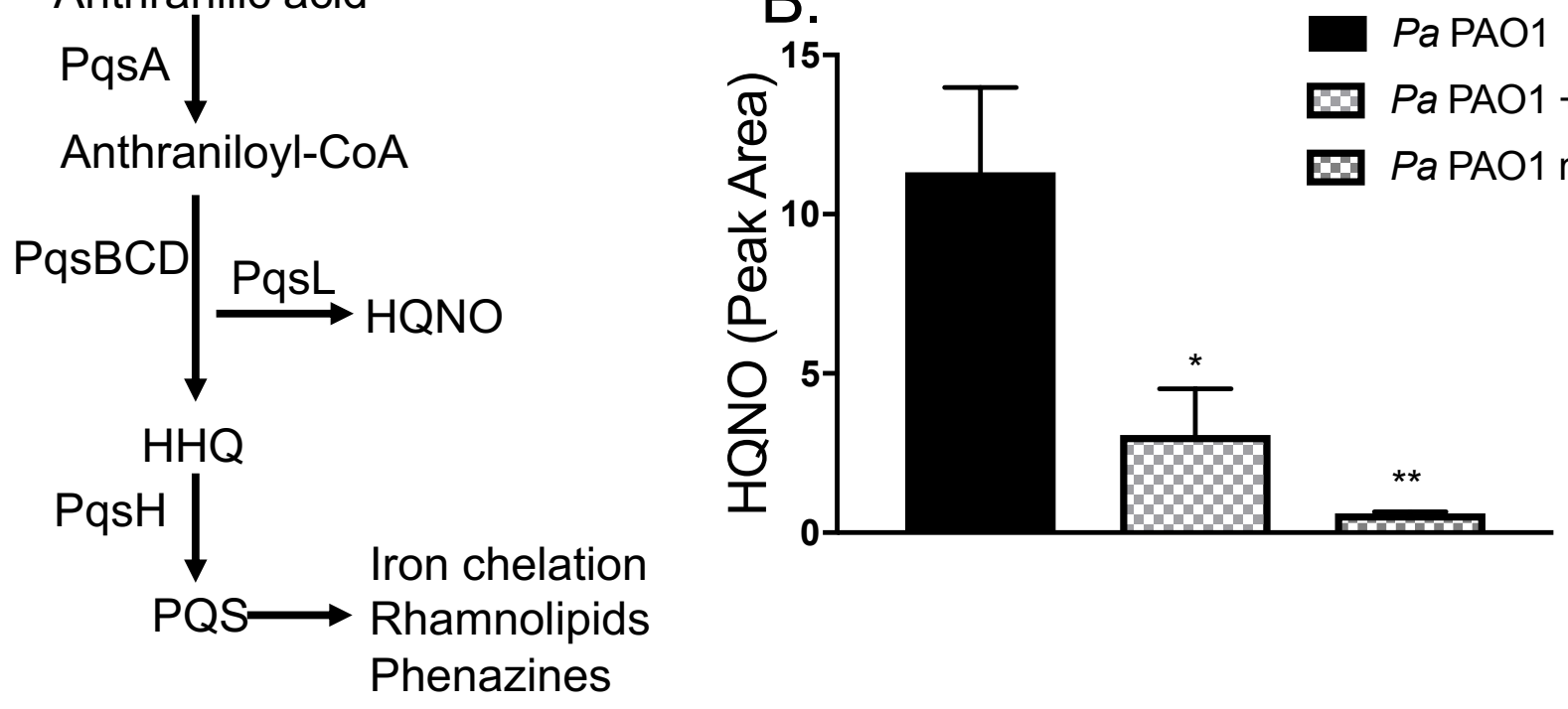

C.

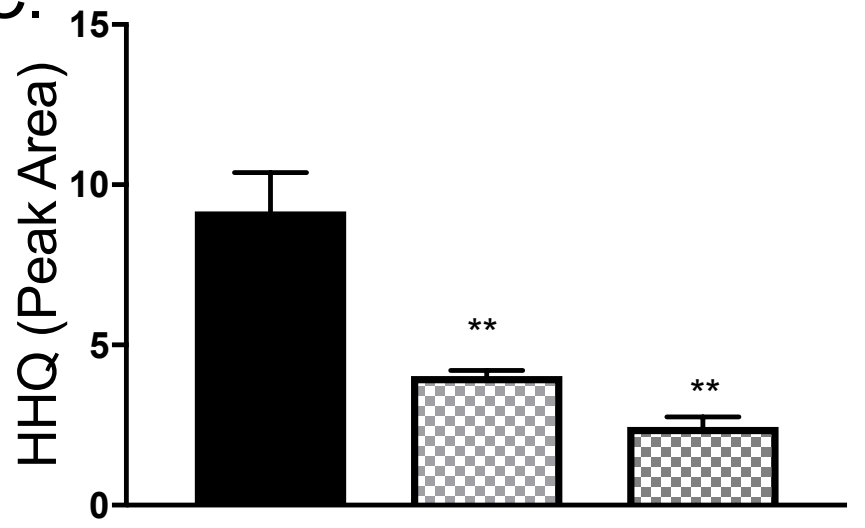

E.

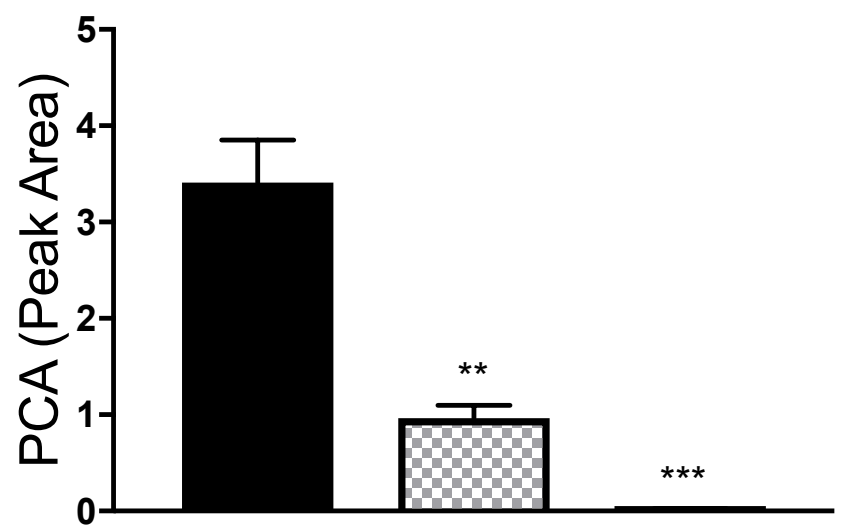

D.

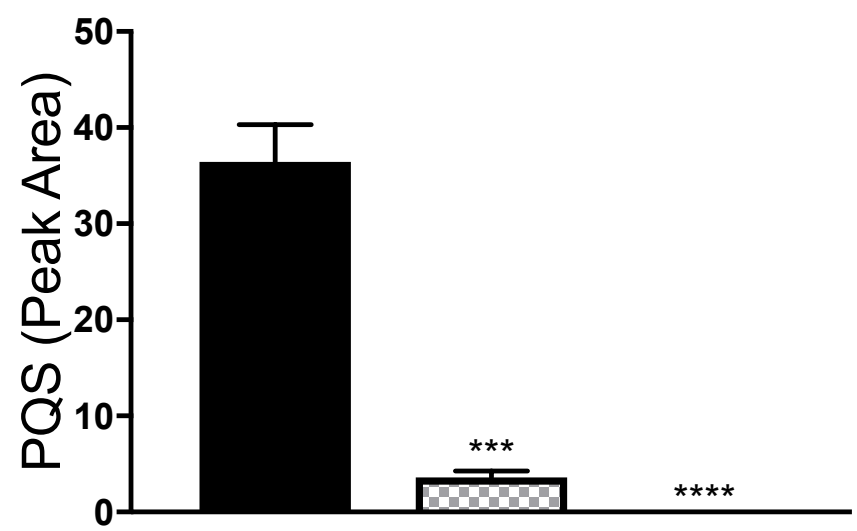

$\mathrm{F}$.

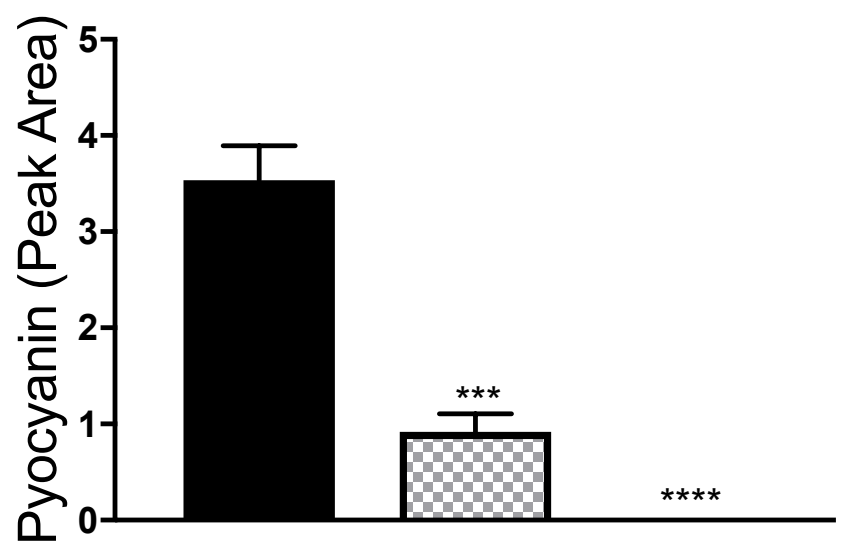


Figure 6. Exogenous alginate downregulates PQS quorum sensing. A) The PQS quorum

sensing regulon. PQS synthesis is dependent on the $p q S A B C D$ genes located in the pqs operon.

Both HHQ and HQNO have direct antimicrobial properties, while PQS is the ligand for PqsR.

When PQS level is high this ligand interacts with PqsR to positively regulate many downstream virulence factors. PQS has direct iron-chelating function and promotes expression of siderophore-encoding genes. The downstream effects listed here focus on effects relevant to this study and are not exhaustive. B-F) In Panel B is shown is the legend for all graphs in this figure. LC-MS/MS was used to quantify B) HQNO C) HHQ D) PQS E) PCA and F) pyocyanin produced by $P$. aeruginosa. 
bioRxiv preprint doi: https://doi.org/10.1101/748459; this version posted August 28, 2019. The copyright holder for this preprint (which was not certified by peer review) is the author/funder, who has granted bioRxiv a license to display the preprint in perpetuity. It is made available under

A.

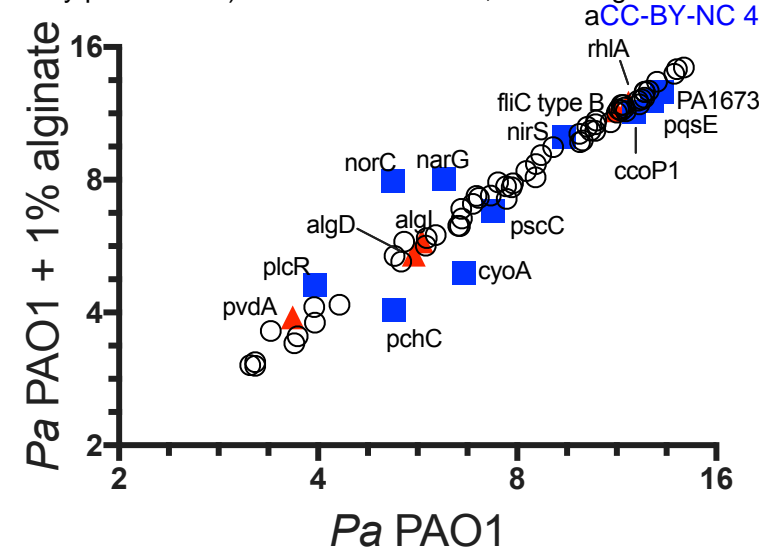

C.

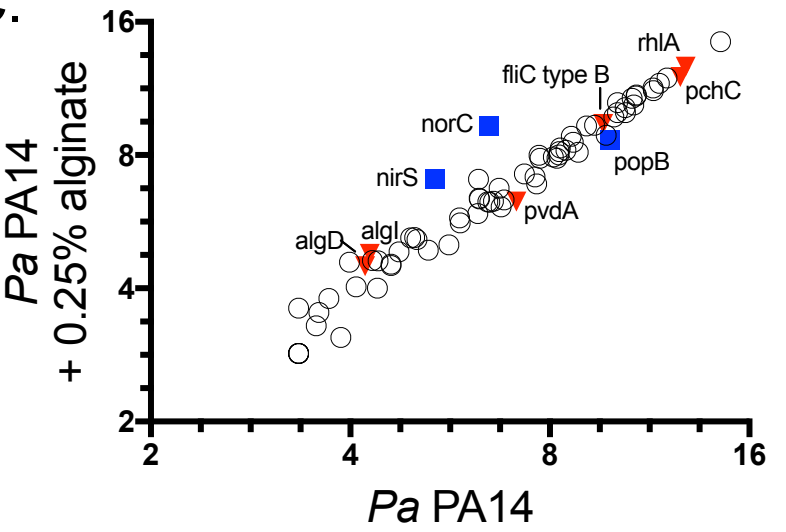

E.

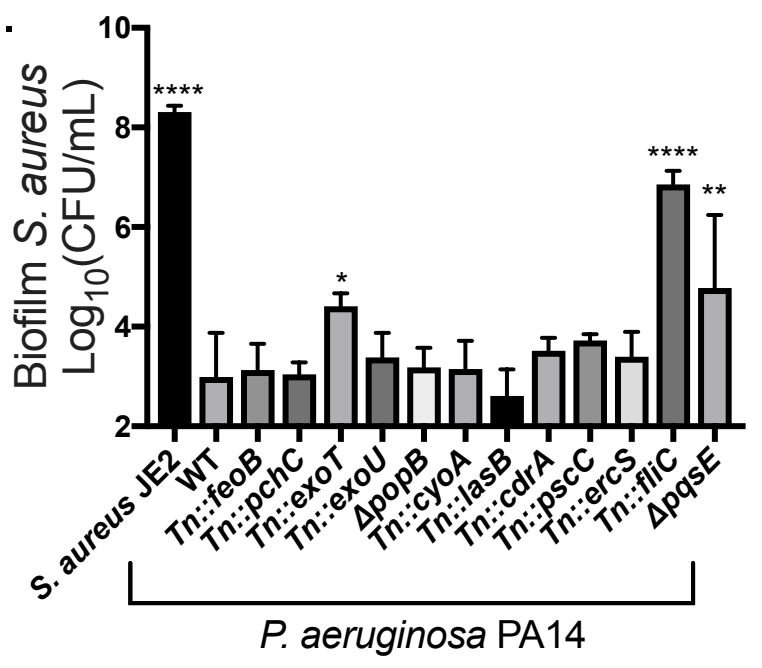

G.

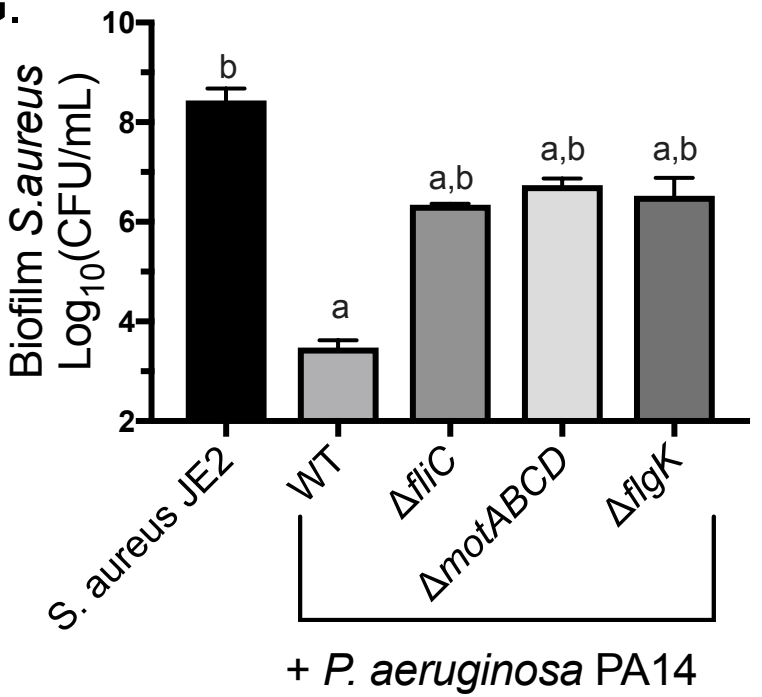

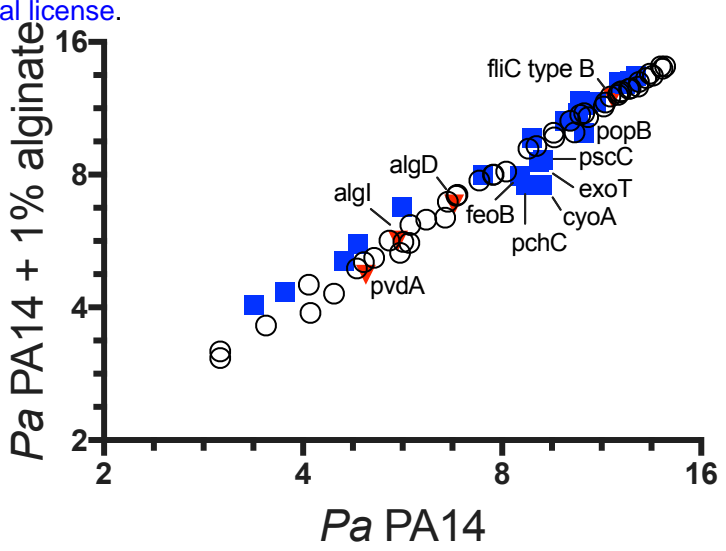

D.

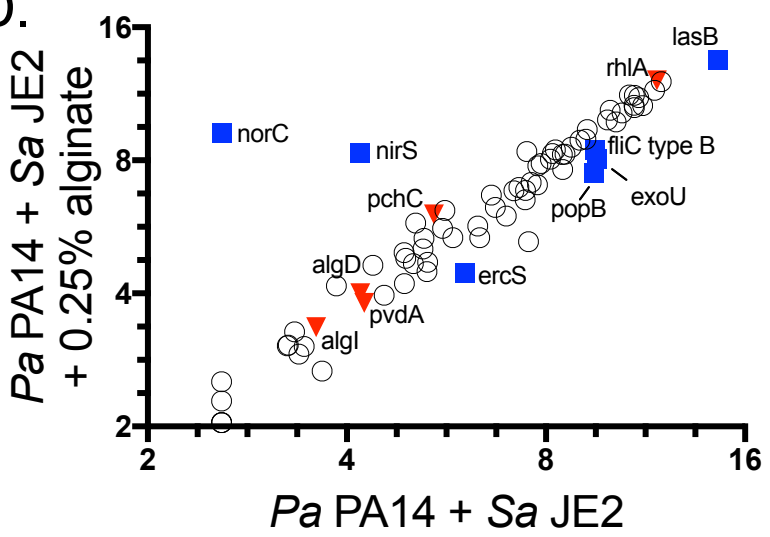

F.

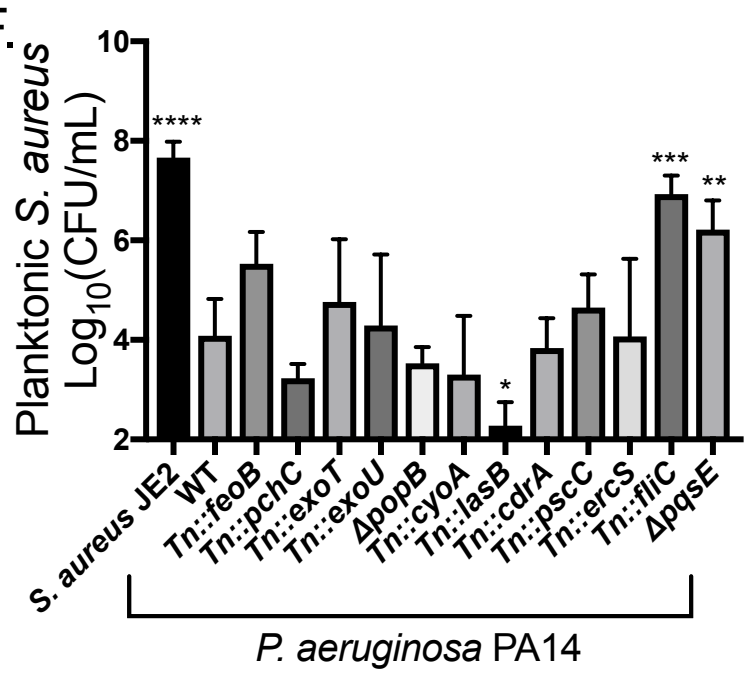

H.

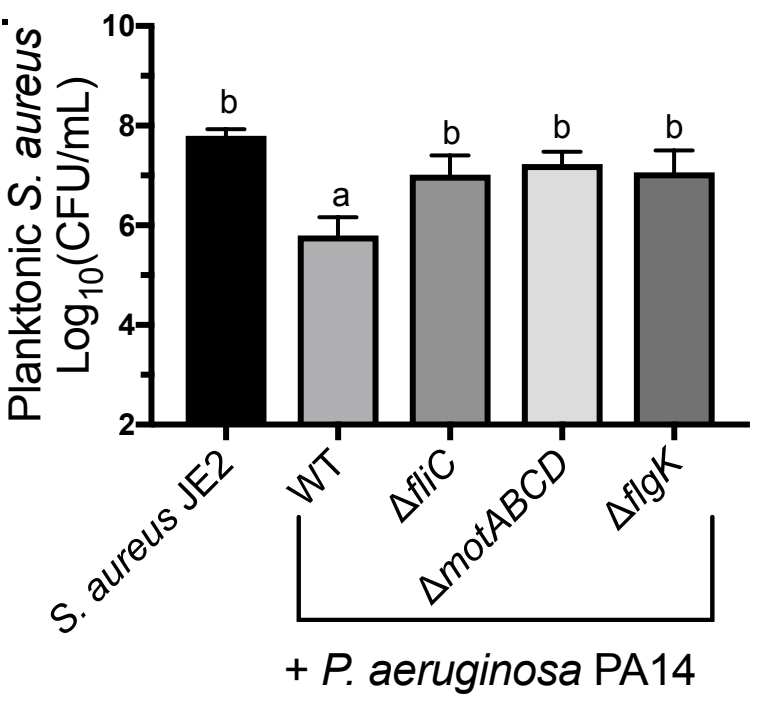


Figure 7. Alginate alters the expression of $P$. aeruginosa genes essential for $S$. aureus killing. A-

D) Raw Nanostring counts were normalized to positive controls and three housekeeping genes

$(r p o D, p p i D, f b p)$ and $\log _{2}$ transformed. Three biological replicates per condition. Significantly

differentially expressed genes were determined by unpaired $t$-test followed by the two-stage

linear step-up procedure of Benjamini, Krieger, and Yekutieli (with $q 1 \%$ for false discovery), and

are marked by blue squares and labeled with the gene name. Genes significantly differentially

expressed by mucoid $P$. aeruginosa in a previous study (32) but not by $P$. aeruginosa in the

presence of exogenous alginate are marked by red triangles. A) P. aeruginosa PAO1 and B) $P$.

aeruginosa PA14 sub-cultured into TSB or TSB $+1 \%$ alginate during mid-log growth phase for 45

minutes. For clarity, only significantly downregulated genes are labeled in B). P. aeruginosa PA14 C)

mono-cultured and D) co-cultured with S. aureus JE2 in TSB $+0.25 \%$ alginate for 8 hours. E-F) S.

aureus JE2 survival in the E) biofilm and F) planktonic fractions after 16 hour co-culture with $P$.

aeruginosa PA14 mutants for genes downregulated in one or more Nanostring experiment.

Significance determined by one-way ANOVA with Dunnett's post-test comparison to S. aureus in

monoculture. ${ }^{*} p<0.05, * * p<0.01, * * * p<0.001, * * * * p<0.0001$. G-H) S. aureus JE2 survival in the

G) biofilm and $\mathbf{H}$ ) planktonic fractions after 16 hour co-culture with P. aeruginosa PA14 motility

mutants. Significance determined by one-way ANOVA with Dunnett's post-test. a, $p<0.05$ with $S$.

aureus JE2 as the reference. $\mathrm{b}, \mathrm{p}<0.05$ with $P$. aeruginosa PAO1 $+S$. aureus JE2 as the reference. 
bioRxiv preprint doi: https://doi.org/10.1101/748459; this version posted August 28,2019 . The copyright holder for this preprint (which was not

A) certified by peer review) is the author/funder, who has granted bioRxiv a license to display the preprint in perpetuity. It is made available under

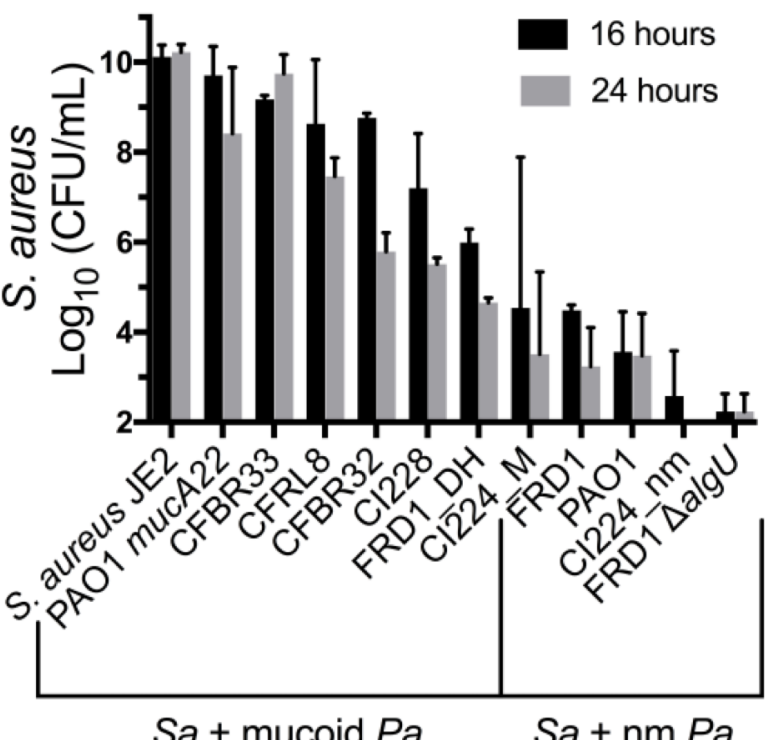

C)

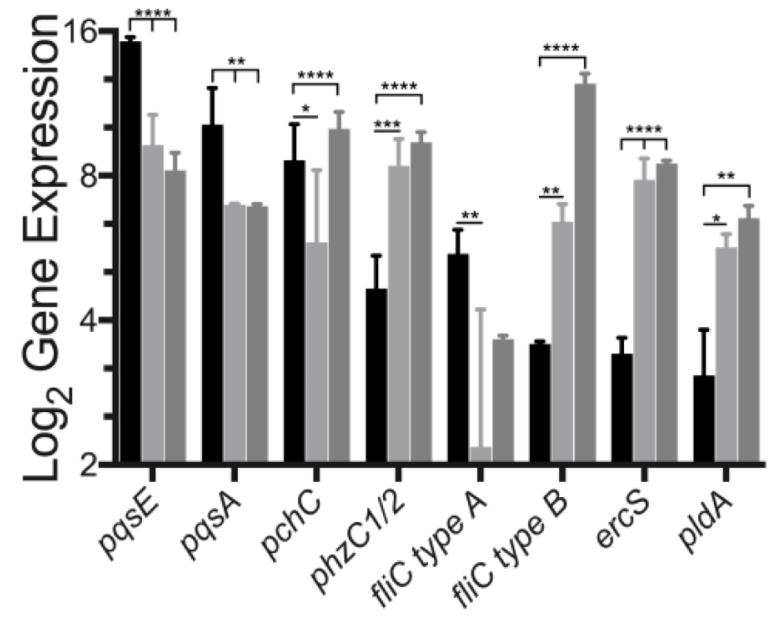

Pa CFBR32
Pa PAO1 mucA22
aCC-BY-NC 4 B $)^{\text {nternational license. }}$

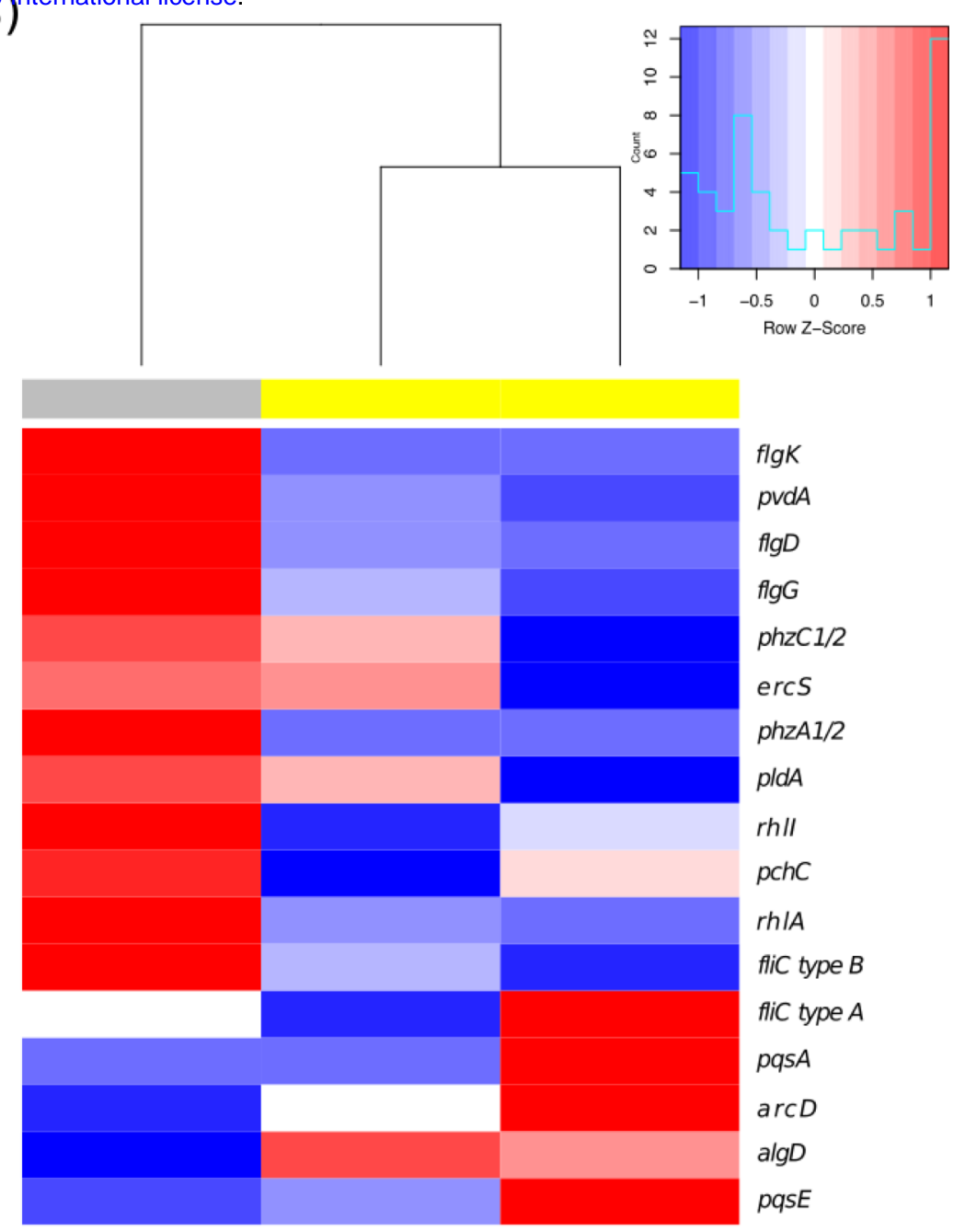

Pa PAO1

\section{高竞}

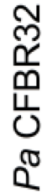


Figure 8. Mucoid clinical isolates have varying effects on S. aureus in coculture. A) S. aureus

JE2 growth after 16 and 24 hours of co-culture in flasks shaking at $225 \mathrm{rpm}$ in TSB with $P$.

aeruginosa clinical isolates. $\mathrm{Nm}=$ nonmucoid. $\mathrm{B}-\mathrm{C}) \log _{2}$ transformation of Nanostring counts normalized to positive controls and three housekeeping genes $(r p o D, p p i D, f b p)$ for the indicated transcripts for clinical isolate $P$. aeruginosa CFBRPA32, mucoid laboratory strain $P$. aeruginosa PAO1 mucA22, and wild-type laboratory strain P. aeruginosa PAO1 after 24 hours culture in flasks shaking at $225 \mathrm{rpm}$ in TSB. Two biological replicates per strain. Gene expression was analyzed by two-way ANOVA followed by Tukey's multiple comparisons. B) Heatmap and dendrogram of all genes significantly differentially regulated between any two strains. Expression values displayed as within-row Z-scores. Yellow indicates mucoid strains, and gray indicates non-mucoid strains. C) All genes significantly differentially regulated between $P$. aeruginosa CFBRPA32 and P. aeruginosa PAO1 mucA22. ${ }^{*} p<0.05,{ }^{*} p<0.01$, $* * * p<0.001, * * * * p<0.0001$ 